\title{
The rate of period change in pulsating DB white dwarf stars
}

\author{
A. H. Córsico ${ }^{1,2, \star}$ and L. G. Althaus ${ }^{1,2,3, \star}$ \\ ${ }^{1}$ Facultad de Ciencias Astronómicas y Geofísicas, Universidad Nacional de La Plata, Paseo del Bosque, s/n, (1900) La Plata, \\ Argentina \\ e-mail: acorsico@fcaglp.unlp.edu.ar \\ 2 Instituto de Astrofísica La Plata, IALP, Conicet, Argentina \\ ${ }_{3}$ Departament de Física Aplicada, Universitat Politècnica de Catalunya, Av. del Canal Olímpic, s/n, 08860 Castelldefels, Spain \\ e-mail: leandro@fa.upc.es
}

Received 29 May 2004 / Accepted 5 August 2004

\begin{abstract}
In this work, we present the theoretically expected rates of pulsation period change for V777 Her (DBV) variable stars. To this end we employ new evolutionary models representative of pulsating DB white dwarf stars computed in a self-consistent way with the predictions of time-dependent element diffusion. At the hot edge of the DB instability strip, the envelopes of the models are characterized by a diffusion-induced double-layered chemical structure. We compute the numerical values of rates of period change by solving the equations of linear, adiabatic, nonradial stellar oscillations. We examine the effects of varying the stellar mass, the mass of the helium envelope and the neutrino emission on the expected period changes. We present extensive tabulations of our results which could be useful for comparison with future detections of the rate of period change in pulsating DB white dwarfs.
\end{abstract}

Key words. dense matter - stars: evolution - stars: white dwarfs - stars: oscillations

\section{Introduction}

V777 Her (or DBV) stars are $g$ (gravity)-mode variable white dwarfs with helium-rich atmospheres (DB) and intermediate effective temperature $\left(T_{\text {eff }} \sim 25000 \mathrm{~K}\right)$, the pulsating nature of which was predicted two decades ago on theoretical grounds by Winget et al. (1982a) and shortly after confirmed observationally by Winget et al. (1982b). Since then, considerable effort has been devoted to studying these stars. In particular, the multiperiodic star GD 358, the most extensively studied member of the DBV class, has been the subject of numerous investigations devoted to disentangling its internal structure and evolution, initially by means of "hand on" asteroseismological procedures (Bradley \& Winget 1994) and later by employing objective fitting techniques (see, e.g., Metcalfe et al. 2000, 2001, 2002). In particular, Metcalfe et al. (2001) - see also Metcalfe et al. (2002) - have recently applied genetic algorithm-based procedures to place constraints on the ${ }^{12} \mathrm{C}(\alpha, \gamma){ }^{16} \mathrm{O}$ reaction rate from inferences for the abundance of central oxygen in GD 358.

As a variable white dwarf cools down, its oscillation periods $(P)$ vary in response to evolutionary changes in the mechanical structure of the star. Specifically, as the temperature in the core of a white dwarf decreases, the plasma increases its degree of degeneracy and the Brunt-Väisälä frequency - the most important physical quantity in $g$-mode pulsations - diminishes,

* Member of the Carrera del Investigador Científico y Tecnológico, CONICET, Argentina. and the pulsational spectrum of the star shifts to longer periods. On the other hand, residual gravitational contraction (if present) acts in the opposite direction, thus shortening the pulsation periods. Competition between the increasingly degeneracy and gravitational contraction gives rise to a detectable temporal rate of change of periods $(\dot{P} \equiv \mathrm{d} P / \mathrm{d} t)$. Roughly, the rate of change of the pulsation period is related to the rates of change of the temperature at the region of the period formation, $\dot{T}$, and of the stellar radius, $\dot{R}_{*}$, by the expression (Winget et al. 1983):

$\frac{\dot{P}}{P} \approx-a \frac{\dot{T}}{T}+b \frac{\dot{R}_{*}}{R_{*}}$

where $a$ and $b$ are constants whose values depend on the details of the white dwarf modeling ( $a, b \approx 1)$. The first term in Eq. (1) corresponds to the rate of change in period induced by the cooling of the white dwarf and it is a positive contribution, while the second term represents the rate of change due to gravitational contraction and it is a negative contribution. In principle, the rate of change of period can be measured by observing a pulsating white dwarf over several seasons when one or more stable pulsation periods are present in their light curves. In the case of pulsating DA (hydrogen-rich atmospheres) white dwarfs - also termed DAV or ZZ Ceti variable stars - cooling dominates over gravitational contraction, in such a way that the second term in Eq. (1) is negligible, and only positive values of the rate of change of period are expected. This is the case of the ZZ Ceti 
G117-B15A, for which Kepler et al. (2000) have quoted a rate of change of the 215.2 s period of $\dot{P}=(2.3 \pm 1.4) \times 10^{-15} \mathrm{~s} \mathrm{~s}^{-1}$. In addition, Mukadam et al. (2003) report that the period at $213.13 \mathrm{~s}$ observed in other ZZ Ceti star, R548, drifts at a rate $\dot{P} \leq(5.5 \pm 1.9) \times 10^{-15} \mathrm{~s} \mathrm{~s}^{-1}$. For the high effective temperatures characterizing the DOV/PNNV instability strip, gravitational contraction is still significant, to such a degree that its influence on $\dot{P}$ can overcome the effects of the cooling. In this case the second term in Eq. (1) is not negligible anymore and consequently positive or negative $\dot{P}$ values are possible. For these hot pre-white dwarfs, what determines that $\dot{P}$ is either positive or negative is the character of the mode: if their pulsation period corresponds to a confined mode ${ }^{1}$ then a positive value of $\dot{P}$ is expected, while if the mode is a trapped one ${ }^{2}$ we would expect a negative $\dot{P}$ value (see Kawaler $\&$ Bradley 1994). For PG 1159-035, the prototype star of the DOV class, a rate of $\dot{P}=(13.07 \pm 0.03) \times 10^{-11} \mathrm{~s} \mathrm{~s}^{-1}$ has been measured for the $516 \mathrm{~s}$ pulsation period, although uncertainties in the modeling of its interior structure have led to ambiguous conclusions (Winget et al. 1985; Costa et al. 1999). As for the DBV stars, in which the influence of the gravitational contraction on $\dot{P}$ is negligible, the expected rate of period change from theoretical evolutionary computations should be positive and of the order of $\sim 10^{-13}-10^{-14} \mathrm{~s} \mathrm{~s}^{-1}$, although detections of $\dot{P}$ have not yet been made in any DBV star.

Observational measurement of $\dot{P}$ provides a sensitive probe of the structure and evolution of white dwarf stars. As shown by Winget et al. (1983), a measurement of the rate of period change of a pulsating white dwarf constitutes, particularly for DAV and DBV white dwarfs (which evolve at almost constant radius) a direct measurement of the cooling rate of the star. This, in turn, provides valuable information about the core chemical composition. Also, measurement of the rate of period change of pulsating white dwarfs in the DAV, DBV and DOV instability strips would allows astronomers to calibrate the cooling sequence age. This, in turn, could be employed to infer the age of the galactic disk in the solar neighborhood (Winget et al. 1987). In addition, the measurement of $\dot{P}$ in variable white dwarfs can be employed to set constraints on particle physics. McGraw et al. (1979) were the first to suggest that hot pulsating white dwarfs could be employed to determine the effect of neutrino cooling as a star becomes a white dwarf (see also Winget et al. 1983). The influence of neutrino energy loss on $\dot{P}$ was discussed in detail by Kawaler et al. (1986) for the case of DBV and DOV white dwarfs. In addition, Isern et al. (1992) - see also Córsico et al. (2001a) - have explored the effect of axion emissivity in DAV stars. Recently, O'Brien \& Kawaler (2000) have discussed the possibility of inferring limits on the theoretically determined plasmon neutrino emission rates by employing DOV white dwarfs.

More recently, D. Winget and collaborators have drawn attention the attractive possibility of employing DBV stars as plasmon neutrino detectors (Winget et al. 2004). These authors

\footnotetext{
1 That is, a mode with their eigenfunctions sensitive to the deep regions of the star.

2 That is, a mode whose eigenfunctions are concentrated mostly toward the outer layers.
}

- see also Kawaler et al. (1986) - have noted that in the hotter region of the DBV instability strip, the plasmon neutrino energy losses are several times larger than the losses due to photon emission. Their results suggest that measurement of the evolutionary period change in hot DB white dwarf stars would constitute an excellent probe of the plasmon neutrino production rates, if period changes in DBV stars could be assessed. The authors discuss several observational strategies to estimate $\dot{P}$ for DBV white dwarfs, in addition to the ongoing observations of the hot DBV EC 20058 by Sullivan et al. (2004).

It is worth noting that the study of Winget et al. (2004) is based on stellar models for pulsating DB stars with a chemical structure characterized by a pure helium envelope atop a carbon-oxygen core, i.e., a single-layered envelope structure. However, on the basis of evolutionary calculations including time-dependent element diffusion, Dehner \& Kawaler (1995) and Gautschy \& Althaus (2002) have found that, if DB white dwarfs descend from PG 1159 stars $^{3}$, their envelopes would be characterized by the presence of a double-layered chemical structure. Indeed, such calculations show that by the time the DB instability strip is reached, models are characterized by two different chemical transition zones, i.e., a double-layered configuration. In fact, above the carbon-oxygen core, there exists an envelope consisting of an intershell region rich in helium, carbon and oxygen, the relics of the short-lived mixing episode occurred during the last helium thermal pulse that leads to the born-again episode, and an overlying pure helium mantle resulting from the gravitational settling of carbon and oxygen. More recently, Fontaine \& Brassard (2002) have demonstrated that the theoretical period spectrum of DBV white dwarf models which incorporate a double-layered envelope, turns out to be markedly distinct from that expected for a single-layered configuration. As shown by these authors, this is particularly important when attempts at constraining the core composition of DBs and the ${ }^{12} \mathrm{C}(\alpha, \gamma){ }^{16} \mathrm{O}$ reaction rate are made. This point has recently been addressed by Metcalfe et al. (2003), who have incorporated both the double-layered envelope feature and adjustable carbon-oxygen cores in DB asteroseismological fittings.

In a recent work, Althaus \& Córsico (2004) have presented new evolutionary models of DB white dwarf stars for various masses of the helium content and several stellar masses, computed in a self-consistent way with the predictions of timedependent element diffusion. The initial outer layer chemical stratification assumed for such models corresponds to that characterizing PG 1159 stars. By the time the domain of the DBVs is reached, the envelopes of these models are characterized by a double-layered chemical structure induced by diffusion. The authors find that, depending on the stellar mass, if DB white dwarf progenitors are formed with a helium content smaller than $\sim 10^{-3} M_{*}$, a single-layered configuration is expected to emerge during the DB pulsation instability strip. As shown in

\footnotetext{
3 PG 1159 stars are hydrogen-deficient post-asymptotic giant branch (post-AGB) stars that are widely believed to be the result of a born-again episode (see Schönberner 1979 and Iben et al. 1983) and the immediate predecessors of most hydrogen-deficient white dwarfs.
} 
that paper, the period spacing diagrams exhibit mode-trapping substructures when a double-layered configuration characterizes the envelope of the models, substructures that are virtually absent in single-layered envelope models.

In view of the Winget et al. (2004)'s claims about the potential of employing DBV white dwarf stars to place constraints on the plasmon neutrino emissivity, we judge that the computation of the rate of period change in the frame of new stellar models which incorporate an updated input physics, particularly timedependent diffusion processes as well as realistic initial envelope chemical stratifications as predicted by the evolutionary history of the progenitor stars, would be worthwhile to be done. This is the aim of the present paper. An additional motivation is the lack of modern tabulations of the rate of period change for DBV stars in the literature. Specifically, we present theoretical values for the rates of pulsation period change for the effective temperature range of interest. This is done by means of several tables providing $P$ and $\dot{P} / P(\equiv \mathrm{d} \ln P / \mathrm{d} t)$ values corresponding to dipole modes $(\ell=1)$ for different stellar masses, effective temperatures and thickness of the helium envelope. DB white dwarf evolutionary models are the same as those presented in Althaus \& Córsico (2004). We compute the numerical values of $\dot{P} / P$ by solving the equations of linear, adiabatic, nonradial stellar oscillation for evolutionary models representing DB white dwarfs. We briefly examine the effect of changing the various structural parameters on the theoretical rate of period change.

The rest of the paper is organized as follow. In Sect. 2 we describe our evolutionary DB white dwarf models, and in Sect. 3 we examine the effects of varying the stellar mass, the mass of the helium envelope and the neutrino emission on the $\dot{P} / P$ values. Finally, we close the paper with a short summary in Sect. 4.

\section{Input physics and evolutionary models}

The evolutionary models employed in this work have been obtained with the DB white dwarf evolutionary code developed at La Plata Observatory. The code is that described in Althaus \& Córsico (2004) and references therein (see also Gautschy \& Althaus 2002). In particular, microphysics includes an updated version of the equation of state of Magni \& Mazzitelli (1979), OPAL radiative opacities for arbitrary metallicity (Iglesias \& Rogers 1996) including carbon- and oxygenrich compositions, and up-to-date neutrino emission rates and conductive opacities. In particular, opacities for various metallicities are required because of the metallicity gradient that develops in the envelopes as a result of gravitational settling. In this work, convection is treated in the framework of the mixing length theory as given by the ML2 parameterization (see Tassoul et al. 1990). Our evolutionary models are calculated self-consistently with the predictions of time-dependent element diffusion. Our treatment of diffusion for multi-component gases includes gravitational settling and chemical and thermal diffusion for the nuclear species ${ }^{4} \mathrm{He},{ }^{12} \mathrm{C}$ and ${ }^{16} \mathrm{O}$. As for the chemical composition of the core, we have adopted the chemical profiles of Salaris et al. (1997).
Our starting stellar configurations correspond to hot white dwarf structures with a realistic outer layer chemical stratification appropriate to that of hydrogen-deficient PG 1159 stars. These stars, presumed to be the direct ancestors of most DB white dwarfs, are widely believed to be the result of a bornagain episode experienced by a post-AGB remnant on the early white dwarf cooling branch. As a result of such an episode, most of the hydrogen content is completely burnt, and the envelope is eventually characterized by an uniform chemical composition of helium, carbon and oxygen. In our models we have assumed, for such compositions, mass fractions of $0.42,0.36$ and 0.22 , respectively, following observed abundance patterns in PG 1159 stars. Also, we have varied the stellar mass in the range $0.50-0.85 M_{\odot}$, and the mass of the helium content to be $M_{\mathrm{He}}: 8 \times 10^{-3}, 9 \times 10^{-4}$ and $1 \times 10^{-4} M_{*}$. With regard to the helium envelope, some words are in order. The mass of the helium envelope is constrained by the theory of post-AGB evolution to be $\lesssim 10^{-2} M_{*}$ (Iben 1989; D’Antona \& Mazitelli 1991). Also, recent full evolutionary calculations predict the helium content to range from $10^{-2} M_{*}$ (for a $0.6 M_{\odot}$ white dwarf remnant, see Herwig et al. 1999) to $10^{-3} M_{*}$ (for a $0.93 M_{\odot}$ white dwarf, see Althaus et al. 2003). Such values are upper limits in the sense that post-AGB mass loss episodes could reduce them considerably. In fact, as recently emphasized by Werner (2001), the existence of mass-loss rates in the range $10^{-7}-10^{-8} M_{\odot} / \mathrm{yr}$ cannot be discounted in many luminous PG 1159 stars. In addition, tentative evidence for the persistence of mass-loss rates of the order $10^{-7}-10^{-10} M_{\odot} / y r$ down to the domain of hot heliumrich white dwarfs has been presented (see Werner 2001). Thus, the existence of hot hydrogen-deficient pre-white dwarf stars with a helium content of the order of $10^{-3} M_{*}$ could not be discounted even in the case of DB white dwarf remnants with masses as low as $0.6 M_{\odot}($ see Althaus \& Córsico 2004).

\section{Rates of period change}

In what follows, we describe the main results of our pulsation calculations. We mostly concentrate on the theoretical rate of period change. We assess the $\dot{P} / P$ values with the help of the pulsational code employed in Córsico et al. (2001b), appropriately modified to study pulsating DB white dwarfs. In particular, the treatment we follow to assess the Brunt-Väisälä frequency $(N)$ is that proposed by Brassard et al. (1991).

We begin by examining Fig. 1, in which a representative spatial run of the Brunt-Väisälä frequency of a DB white dwarf is displayed. The model is characterized by a stellar mass of $0.60 M_{\odot}$, an effective temperature of $\approx 25300 \mathrm{~K}$ and a helium content of $M_{\mathrm{He}} \approx 9 \times 10^{-4} M_{*}$. In addition, the plot shows the internal chemical stratification of the white dwarf model, and for illustrative purposes the profile of the Ledoux term $B$ (inset), an important quantity related to the computation of the Brunt-Väisälä frequency. The figure emphasizes the role of the chemical interfaces on the shape of the BruntVäisälä frequency. In fact, each chemical transition region produces clear and distinctive features in $N$, which eventually are responsible for the mode trapping properties of the model. At the core region, the dominant feature at $\log q \approx-0.4(q \equiv 1-$ $\left.M_{r} / M_{*}\right)$ is the result of the steep variation of the oxygen/carbon 
Table 1. Periods and relative rates of period change $(\ell=1$ modes $)$ for selected models with $M_{*}=0.50 M_{\odot}$ and $M_{\mathrm{He}}=8 \times 10^{-3} M_{*}$.

\begin{tabular}{|c|c|c|c|c|c|c|c|c|c|c|c|c|c|c|}
\hline \multirow[b]{2}{*}{$k$} & \multicolumn{2}{|c|}{$T_{\text {eff }}=28093 \mathrm{~K}$} & \multicolumn{2}{|c|}{$\overline{227029 \mathrm{~K}}$} & \multicolumn{2}{|c|}{$26026 \mathrm{~K}$} & \multicolumn{2}{|c|}{$25087 \mathrm{~K}$} & \multicolumn{2}{|c|}{$24025 \mathrm{~K}$} & \multicolumn{2}{|c|}{$23031 \mathrm{~K}$} & \multicolumn{2}{|c|}{$22099 \mathrm{~K}$} \\
\hline & $P[\mathrm{~s}]$ & $\dot{P} / P$ & $P[\mathrm{~s}]$ & $\dot{P} / P$ & $P[\mathrm{~s}]$ & $\dot{P} / P$ & $P[\mathrm{~s}]$ & $\dot{P} / P$ & $P[\mathrm{~s}]$ & $\dot{P} / P$ & $P[\mathrm{~s}]$ & $\dot{P} / P$ & $P[\mathrm{~s}]$ & $\dot{P} / P$ \\
\hline 1 & 127.35 & 6.90 & 130.83 & 5.89 & 134.29 & 4.77 & 137.75 & 3.97 & 141.90 & 3.18 & 146.07 & 2.56 & 150.27 & 2.06 \\
\hline 2 & 164.92 & 6.16 & 169.04 & 5.49 & 173.22 & 4.52 & 177.50 & 3.85 & 182.76 & 3.16 & 188.13 & 2.57 & 193.59 & 2.07 \\
\hline 3 & 214.27 & 5.13 & 218.53 & 4.18 & 222.68 & 3.44 & 226.77 & 2.85 & 231.67 & 2.31 & 236.60 & 1.88 & 241.61 & 1.53 \\
\hline 4 & 245.71 & 6.04 & 251.85 & 5.51 & 258.27 & 4.70 & 264.99 & 4.07 & 273.38 & 3.38 & 282.02 & 2.76 & 290.83 & 2.23 \\
\hline 5 & 293.05 & 5.98 & 299.88 & 4.90 & 306.60 & 4.04 & 313.25 & 3.35 & 321.17 & 2.68 & 329.06 & 2.14 & 336.92 & 1.70 \\
\hline 6 & 320.01 & 5.85 & 327.78 & 5.35 & 336.02 & 4.64 & 344.67 & 4.05 & 355.52 & 3.37 & 366.75 & 2.76 & 378.23 & 2.23 \\
\hline 7 & 379.07 & 5.98 & 387.78 & 4.81 & 396.12 & 3.81 & 404.04 & 3.04 & 413.00 & 2.29 & 421.32 & 1.71 & 429.06 & 1.28 \\
\hline 8 & 409.50 & 6.67 & 420.72 & 5.95 & 432.38 & 5.05 & 444.31 & 4.28 & 458.69 & 3.39 & 472.61 & 2.55 & 485.12 & 1.77 \\
\hline 9 & 462.64 & 6.41 & 473.88 & 5.02 & 483.93 & 3.55 & 492.45 & 2.53 & 500.82 & 1.67 & 508.20 & 1.30 & 516.47 & 1.29 \\
\hline 10 & 490.95 & 5.46 & 502.26 & 5.12 & 513.76 & 4.18 & 525.43 & 3.53 & 539.47 & 2.82 & 553.27 & 2.21 & 566.79 & 1.75 \\
\hline 11 & 543.60 & 5.05 & 552.83 & 3.25 & 559.08 & 1.81 & 564.57 & 1.63 & 573.14 & 1.88 & 585.02 & 2.02 & 599.78 & 1.91 \\
\hline 12 & 576.56 & 3.31 & 585.17 & 3.52 & 595.89 & 3.62 & 608.53 & 3.43 & 624.89 & 2.88 & 641.37 & 2.27 & 657.00 & 1.69 \\
\hline 13 & 617.42 & 4.19 & 628.03 & 3.85 & 640.11 & 3.67 & 653.51 & 3.34 & 670.48 & 2.77 & 687.31 & 2.13 & 702.49 & 1.49 \\
\hline 14 & 642.58 & 4.99 & 658.03 & 5.55 & 675.76 & 4.99 & 694.27 & 4.25 & 716.24 & 3.24 & 735.25 & 2.03 & 749.26 & 1.28 \\
\hline 15 & 693.53 & 5.80 & 709.33 & 4.88 & 724.50 & 3.75 & 738.11 & 2.73 & 751.13 & 1.68 & 763.02 & 1.57 & 780.68 & 1.93 \\
\hline 16 & 729.80 & 6.40 & 748.73 & 5.54 & 767.12 & 4.24 & 782.56 & 2.77 & 796.58 & 1.84 & 811.81 & 1.84 & 830.30 & 1.72 \\
\hline 17 & 782.04 & 6.04 & 798.52 & 4.06 & 808.98 & 1.88 & 817.49 & 1.94 & 834.90 & 2.71 & 858.10 & 2.51 & 881.87 & 1.94 \\
\hline 18 & 812.32 & 5.64 & 829.62 & 4.30 & 845.65 & 3.62 & 863.71 & 3.47 & 887.20 & 2.89 & 909.82 & 2.10 & 928.68 & 1.37 \\
\hline 19 & 853.97 & 3.16 & 863.25 & 2.61 & 877.70 & 3.69 & 898.09 & 3.83 & 925.15 & 3.19 & 950.44 & 2.17 & 971.12 & 1.56 \\
\hline 20 & 888.62 & 2.95 & 904.44 & 4.55 & 925.04 & 4.26 & 946.24 & 3.48 & 968.49 & 2.19 & 984.81 & 1.44 & 1006.19 & 1.88 \\
\hline 21 & 930.53 & 5.49 & 952.90 & 5.33 & 976.07 & 4.29 & 996.29 & 2.83 & 1011.97 & 1.43 & 1029.22 & 1.83 & 1054.24 & 1.89 \\
\hline 22 & 965.48 & 6.37 & 990.90 & 5.68 & 1015.89 & 4.33 & 1035.78 & 2.62 & 1057.17 & 2.50 & 1084.83 & 2.38 & 1113.16 & 1.84 \\
\hline
\end{tabular}

Note - All $\dot{P} / P$ values are in units of $10^{-16} \mathrm{~s}^{-1}$.

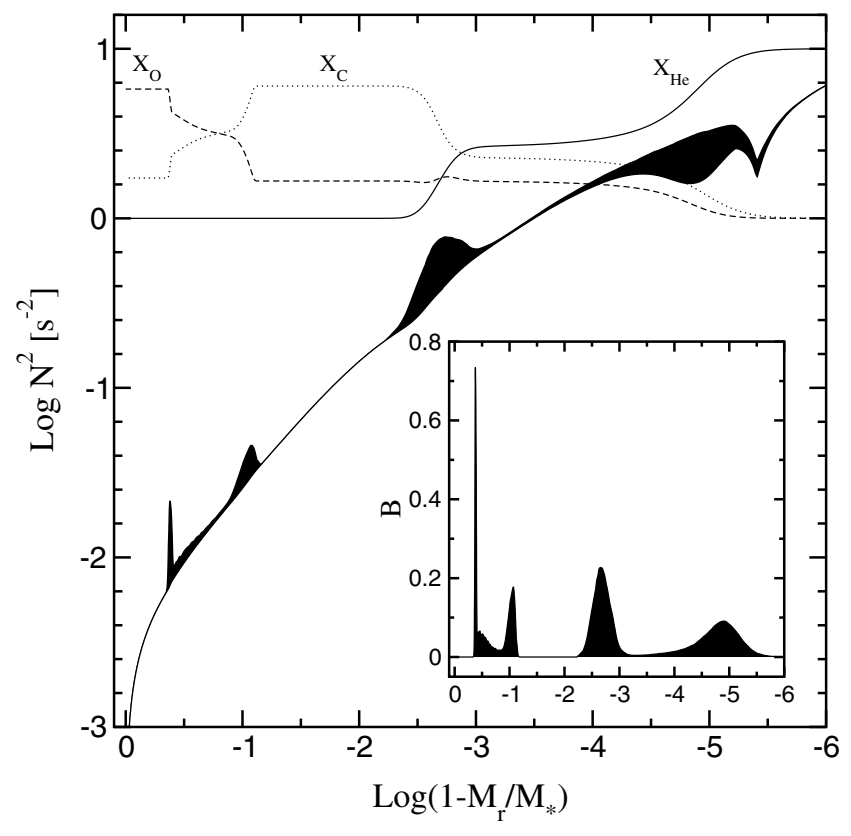

Fig. 1. The run of the squared Brunt-Väisälä frequency in terms of the outer mass fraction, corresponding to a $0.60-M_{\odot}$ DB white dwarf model at $T_{\text {eff }}=25338 \mathrm{~K}$ with a helium content of $M_{\mathrm{He}} \approx 9 \times 10^{-4} M_{*}$. Dark regions denote the contributions of the Ledoux term $B$ (shown in the inset) to the Brunt-Väisälä frequency. The chemical profile is displayed in the upper zone of the plot.

profile. This feature causes strong trapping of certain modes in the core region - "core-trapped" modes; see Althaus et al. (2003) in the context of massive DA white dwarf models. Such modes are characterized by an unusually large oscillation kinetic energy. The less pronounced bump in $N$ at $\log q \approx-1$ is much less relevant to the structure of the period spectrum. Finally, in the envelope of the model we find a double-layered chemical structure. Despite the fact that this structure is modeled by diffusion - and as such, characterized by a very smooth functional shape - its influence on the mode trapping properties is by no means negligible. Indeed, Althaus \& Córsico (2004) have found that the double-layered configuration is responsible for substructures in the period-spacing diagrams. Finally, the steep drop in the Brunt-Väisälä frequency at $\log q \approx-5.5$ is caused by the opacity change due to the metallicity gradient induced by diffusion in the outer layers. This feature occurs close enough to the stellar surface and has not appreciable effects on the model period spectrum.

In this work the rate of period change is estimated as simple differencing of the periods of successive models in each evolutionary sequence. We present $P$ and $\dot{P} / P$ values corresponding to a modest parametric survey of the DB evolutionary models presented in Althaus \& Córsico (2004). In Tables 1 to 9 we provide a set of $P$ and $\dot{P} / P$ theoretical values corresponding to dipole modes $(\ell=1)$ for models with several stellar masses, effective temperatures and thickness of the helium envelope. We feel that these tables could be useful for comparison with future observations of the rate of period change in pulsating DB white dwarfs. Here, we show a sample of our rate of period change values. A more comprehensive tabulation is available at our website ${ }^{4}$.

\footnotetext{
${ }^{4}$ http://www. fcaglp.unlp.edu.ar/evolgroup/
} 
Table 2. Periods and relative rates of period change $(\ell=1$ modes $)$ for selected models with $M_{*}=0.60 M_{\odot}$ and $M_{\mathrm{He}}=8 \times 10^{-3} M_{*}$.

\begin{tabular}{|c|c|c|c|c|c|c|c|c|c|c|c|c|c|c|}
\hline & \multicolumn{2}{|c|}{$T_{\text {eff }}=27925 \mathrm{~K}$} & \multicolumn{2}{|c|}{$26938 \mathrm{~K}$} & \multicolumn{2}{|c|}{$26004 \mathrm{~K}$} & \multicolumn{2}{|c|}{$24950 \mathrm{~K}$} & \multicolumn{2}{|c|}{$23966 \mathrm{~K}$} & \multicolumn{2}{|c|}{$23049 \mathrm{~K}$} & \multicolumn{2}{|c|}{$22064 \mathrm{~K}$} \\
\hline$k$ & $P[\mathrm{~s}]$ & $\dot{P} / P$ & $P[\mathrm{~s}]$ & $\dot{P} / P$ & $P[\mathrm{~s}]$ & $\dot{P} / P$ & $P[\mathrm{~s}]$ & $\dot{P} / P$ & $P[\mathrm{~s}]$ & $\dot{P} / P$ & $P[\mathrm{~s}]$ & $\dot{P} / P$ & $P[\mathrm{~s}]$ & $\dot{P} / P$ \\
\hline 1 & 123.16 & 4.95 & 126.25 & 4.05 & 129.36 & 3.30 & 133.16 & 2.56 & 137.01 & 2.00 & 140.92 & 1.59 & 145.61 & 1.26 \\
\hline 2 & 159.46 & 4.93 & 163.45 & 4.05 & 167.49 & 3.30 & 172.39 & 2.55 & 77.33 & 1.98 & 182.30 & 1.55 & 188.12 & 1.19 \\
\hline 3 & 197.64 & 3.55 & 201.20 & 2.95 & 204.81 & 2.42 & 209.21 & 89 & 70 & 1.49 & & 1.17 & 223.44 & 0.89 \\
\hline 4 & 235.69 & 5.07 & 241.75 & 4.17 & 247.89 & 3.37 & 255.26 & 2.58 & 262.56 & 1.95 & 269.69 & 1.47 & 277.76 & 1.10 \\
\hline 5 & 271.80 & 3.81 & 277.04 & 3.15 & 282.33 & 2. & 288.72 & .99 & 95.23 & 1.57 & 301.91 & 1.26 & 310.08 & 1.04 \\
\hline 6 & 307.31 & 5.00 & 315.09 & 4.10 & 322.93 & 3.30 & 332.32 & 2.52 & 41.62 & 1.91 & 350.68 & 1.44 & 360.76 & 1.04 \\
\hline 7 & 34 & 3.24 & 35 & 2.60 & 35 & 2. & & 1.61 & & 1.26 & & 1.01 & & 0.82 \\
\hline 8 & 394.66 & 4.56 & 403.12 & 3.28 & 410.34 & 2.24 & 417.58 & 1.44 & 423.93 & 1.03 & 430.12 & 0.82 & 437.94 & 0.72 \\
\hline 9 & 419.51 & 2.96 & 426.33 & 2.81 & 434.42 & & 445 & 0 & 4 & 2.02 & 471 & 1.63 & 487.89 & 1.25 \\
\hline 10 & 460.04 & 3.62 & 468.28 & 2.90 & 476.48 & 2.37 & 486.64 & 1.90 & 497 & 1.52 & 507.85 & .17 & 519.73 & 0.86 \\
\hline 11 & 486.33 & 4.01 & 496.93 & 3.68 & 508.45 & 3.1 & 522.89 & 2.49 & 5 & 1.84 & 55 & 1.26 & 56 & 0.86 \\
\hline 12 & 533.62 & 3.99 & 544.07 & 3.11 & 553.83 & 33 & 564.13 & 1.52 & 5 & 1.04 & & 0.93 & & 1.00 \\
\hline 13 & 569.46 & 4.28 & 581.40 & 3.3 & 592.27 & & 604 & 1. & 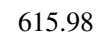 & 1.41 & 628.86 & 1.20 & 645.01 & 0.99 \\
\hline 14 & 608.5 & 3.52 & 618.1 & 2.4 & 627 & 2. & 640 & 1.99 & 0 & 1.75 & 672.03 & 1.35 & 689.79 & 0.98 \\
\hline 15 & 632.97 & 3.30 & 644.83 & 3.29 & 658.72 & 3.01 & 676 & 2.44 & 69 & 1.81 & 711.66 & 1.23 & 729.36 & 1.01 \\
\hline 16 & 673.56 & 3.12 & 685.30 & 2.98 & 698.24 & & 713.68 & 1.88 & 727.50 & 1.26 & 740.44 & 1.04 & 759.67 & 1.13 \\
\hline 17 & 715.7 & 4.68 & 732.38 & 3.6 & 747.30 & & 761.23 & 1. & 773.06 & 1.13 & 787.15 & 1.10 & 807.43 & 1.10 \\
\hline 18 & & 4.03 & 767.22 & 2.9 & 780.23 & 2. & 796.5 & 1. & 815.98 & 1.76 & 836.16 & 1.34 & 858.69 & 1.09 \\
\hline 19 & 787.73 & 4.01 & 801.9 & 2.74 & 815.41 & 2.34 & 834.17 & 2.15 & 89 & 1.72 & 874.52 & 1.21 & 897.69 & 1.17 \\
\hline 20 & 818.66 & 2.76 & 831.58 & 2.88 & 848.17 & 2.87 & 870.67 & 2.33 & 892.11 & 1.59 & 910.63 & 1.12 & 935.40 & 1.22 \\
\hline 21 & 856.30 & 3.36 & 872.61 & 3.2 & 890.45 & & & 1. & 927.66 & 1.28 & 946.28 & 1.23 & 974.56 & 1.29 \\
\hline 22 & & 4.22 & & & & & & & & & & 1.25 & & 1.22 \\
\hline 23 & 935.04 & 4.75 & 956.72 & 3.60 & & 2. & 996.68 & 1.9 & 1020.37 & 1.67 & 1042.83 & 1.13 & 1068.49 & 1.18 \\
\hline 24 & 970.30 & 3.70 & 986.29 & 2.48 & 1002.22 & 2.41 & 1027.69 & 2.41 & 1056.14 & 1.89 & 1082.72 & 1.32 & 1115.12 & 1.33 \\
\hline
\end{tabular}

Note - All $\dot{P} / P$ values are in units of $10^{-16} \mathrm{~s}^{-1}$.

Table 3. Periods and relative rates of period change $(\ell=1$ modes $)$ for selected models with $M_{*}=0.60 M_{\odot}$ and $M_{\mathrm{He}}=9 \times 10^{-4} M_{*}$.

\begin{tabular}{|c|c|c|c|c|c|c|c|c|c|c|c|c|c|c|}
\hline & \multicolumn{2}{|c|}{$T_{\text {eff }}=27991 \mathrm{~K}$} & \multicolumn{2}{|c|}{$26991 \mathrm{~K}$} & \multicolumn{2}{|c|}{$26050 \mathrm{~K}$} & \multicolumn{2}{|c|}{$24994 \mathrm{~K}$} & \multicolumn{2}{|c|}{$24012 \mathrm{~K}$} & \multicolumn{2}{|c|}{$23095 \mathrm{~K}$} & \multicolumn{2}{|c|}{$21967 \mathrm{~K}$} \\
\hline$k$ & $P[\mathrm{~s}]$ & $\dot{P} / P$ & $P[\mathrm{~s}]$ & $\dot{P} / P$ & $P[\mathrm{~s}]$ & $\dot{P} / P$ & $P[\mathrm{~s}]$ & $\dot{P} / P$ & $P[\mathrm{~s}]$ & $\dot{P} / P$ & $P[\mathrm{~s}]$ & $\dot{P} / P$ & $P[\mathrm{~s}]$ & $\dot{P} / P$ \\
\hline 1 & 128.30 & 6.30 & 132.04 & 5.16 & 135.81 & 4.18 & 140.36 & 3.22 & 144.93 & 2.46 & 149.52 & 1.90 & 155.73 & 1.40 \\
\hline 2 & 158.94 & 4.37 & 2.16 & 3.64 & 165.43 & 99 & 9.45 & 2.38 & 73.58 & 1.87 & 7.82 & 1.48 & 33.70 & 1.13 \\
\hline 3 & 198.08 & 5.46 & 03.13 & 4.56 & 208.29 & 3.75 & 4.58 & 2.93 & 220.95 & 2.26 & 227.35 & 1.73 & 235.78 & 1.23 \\
\hline 4 & 237.93 & 4.39 & 2.76 & 3.63 & 247.63 & 2.96 & 3.49 & 2.29 & 59.34 & 1.75 & 265.14 & 1.35 & 272.88 & 1.00 \\
\hline 5 & 269.82 & 5.36 & 6.59 & 4.50 & 283.53 & 71 & 2.03 & 2.91 & 300.68 & 2.26 & 309.46 & 1.76 & 321.30 & 1.29 \\
\hline 6 & 311.14 & 5.09 & 18.40 & 4.13 & 325.56 & 28 & 333.93 & 2.45 & 342.04 & 1.82 & 349.87 & 1.36 & 359.91 & 0.95 \\
\hline 7 & 349.21 & 3.97 & 5.58 & 3.25 & 361.91 & 2.63 & 369.55 & 2.06 & 377.31 & 1.61 & 385.15 & 1.26 & 395.61 & 0.92 \\
\hline 8 & 391.23 & 5.22 & 400.40 & 4.08 & 408.98 & 04 & 418.16 & 2.05 & 426.17 & 1.40 & 433.64 & 1.06 & 444.20 & 0.88 \\
\hline 9 & 424.75 & 3.03 & 0.64 & 2.52 & 437.02 & 32 & 12 & 2.19 & 57.04 & .99 & 469.50 & 1.70 & 487.40 & 1.29 \\
\hline 10 & 454.75 & 4.40 & 4.34 & 3.84 & 474.39 & 22 & 486.79 & 2.55 & 499.33 & 1.96 & 511.58 & 1.45 & 526.52 & 0.93 \\
\hline 11 & 493.48 & 4.12 & 3.12 & 3.54 & 19 & & 63 & 2.36 & 538.02 & 1.76 & 549.65 & 1.26 & 564.25 & 0.93 \\
\hline 12 & 527.52 & 4.81 & 9.51 & 4.08 & 551.55 & 25 & 565.21 & 2.29 & 577.13 & 1.51 & 587.84 & 3 & 603.96 & 1.04 \\
\hline 13 & 576.74 & 4.63 & 7.92 & 3.2 & 596.97 & & 606.11 & 1.49 & 616.36 & 1.44 & 629.52 & 1.40 & 650.19 & 1.16 \\
\hline 14 & 603.77 & 4.09 & 615.16 & 3.41 & 627.28 & 3.02 & 643.50 & 2.60 & 660.97 & 2.09 & 678.25 & 1.52 & 698.82 & 1.02 \\
\hline 15 & 638.71 & 3.32 & 648.61 & 2.88 & 659.84 & 272 & 675.37 & 2.38 & 692.07 & 1.89 & 708.57 & 1.42 & 731.31 & 1.19 \\
\hline 16 & 675.30 & 3.8 & 8.87 & 3.7 & 703.85 & & 721.53 & 2.32 & 736.43 & 1.42 & 748.60 & 0.99 & 768.41 & 1.12 \\
\hline 17 & 715.66 & 5.0 & 2.33 & 4.1 & 748.03 & & 763.80 & 1.86 & 777.88 & 1.47 & 794.60 & 1.40 & 821.62 & 1.28 \\
\hline 18 & 753.44 & 4.6 & 8.98 & 3. & 782.79 & & 90 & 2.1 & 818.05 & 1.95 & 839.09 & & 867.38 & 1.25 \\
\hline 19 & 786.84 & 4.59 & 801.97 & 3.21 & 815.80 & 2.61 & 834.84 & 2.43 & 856.35 & 1.98 & 877.28 & 1.41 & 905.36 & 1.27 \\
\hline 20 & 821.97 & 2.86 & 833.66 & 2.84 & 849.31 & 3.05 & 871.74 & 2.62 & 894.04 & 1.86 & 913.54 & 1.26 & 943.14 & 1.34 \\
\hline 21 & 861.94 & 3.76 & 3.81 & 3.6 & 897.11 & & 917.38 & 2.02 & 933.75 & 1.29 & 951.07 & 1.27 & 984.13 & 1.40 \\
\hline 22 & 900.8 & 4.9 & O & 4. & & & 962.10 & 1.8 & 980.79 & 1.61 & 1002.96 & 1. & 1036.37 & 1.35 \\
\hline 23 & 932.29 & 4.99 & 0.01 & 3.9 & 972.99 & & 995.86 & 2.42 & 1022.65 & 2.13 & 1050.03 & & 1085.76 & 1.38 \\
\hline 24 & 975.02 & 4.44 & 992.10 & 2.77 & 1006.48 & 2.25 & 1028.72 & 2.40 & 1055.00 & 1.95 & 1080.16 & 1.39 & 1118.25 & 1.44 \\
\hline
\end{tabular}

Note - All $\dot{P} / P$ values are in units of $10^{-16} \mathrm{~s}^{-1}$. 
Table 4. Periods and relative rates of period change $(\ell=1$ modes $)$ for selected models with $M_{*}=0.60 M_{\odot}$ and $M_{\mathrm{He}}=1 \times 10^{-4} M_{*}$.

\begin{tabular}{|c|c|c|c|c|c|c|c|c|c|c|c|c|c|c|}
\hline \multirow[b]{2}{*}{$k$} & \multicolumn{2}{|c|}{$T_{\text {eff }}=27956 \mathrm{~K}$} & \multicolumn{2}{|c|}{$26901 \mathrm{~K}$} & \multicolumn{2}{|c|}{$25907 \mathrm{~K}$} & \multicolumn{2}{|c|}{$24969 \mathrm{~K}$} & \multicolumn{2}{|c|}{$24086 \mathrm{~K}$} & \multicolumn{2}{|c|}{$23095 \mathrm{~K}$} & \multicolumn{2}{|c|}{$22021 \mathrm{~K}$} \\
\hline & $P[\mathrm{~s}]$ & $\dot{P} / P$ & $P[\mathrm{~s}]$ & $\dot{P} / P$ & $P[\mathrm{~s}]$ & $\dot{P} / P$ & $P[\mathrm{~s}]$ & $\dot{P} / P$ & $P[\mathrm{~s}]$ & $\dot{P} / P$ & $P[\mathrm{~s}]$ & $\dot{P} / P$ & $P[\mathrm{~s}]$ & $\dot{P} / P$ \\
\hline 1 & 128.01 & 6.60 & 131.90 & 5.43 & 135.85 & 4.42 & 139.85 & 3.56 & 143.90 & 2.84 & 148.82 & 2.18 & 154.65 & 1.62 \\
\hline 2 & 165.08 & 4.84 & 168.74 & 3.99 & 172.45 & 3.27 & 176.21 & 2.65 & 180.02 & 2.14 & 184.64 & 1.64 & 190.08 & 1.23 \\
\hline 3 & 196.61 & 4.98 & 201.14 & 4.18 & 205.84 & 3.48 & 210.68 & 2.87 & 215.66 & 2.34 & 221.74 & 1.80 & 228.90 & 1.33 \\
\hline 4 & 235.43 & 5.20 & 241.07 & 4.32 & 246.82 & 3.54 & 252.65 & 2.86 & 258.49 & 2.27 & 265.43 & 1.69 & 273.34 & 1.21 \\
\hline 5 & 276.42 & 5.28 & 283.08 & 4.31 & 289.75 & 3.47 & 296.38 & 2.75 & 302.94 & 2.17 & 310.74 & 1.63 & 319.88 & 1.22 \\
\hline 6 & 310.29 & 4.75 & 316.96 & 3.86 & 323.68 & 3.14 & 330.44 & 2.53 & 337.28 & 2.05 & 345.66 & 1.60 & 355.69 & 1.21 \\
\hline 7 & 350.48 & 4.94 & 358.32 & 3.98 & 366.00 & 3.14 & 373.48 & 2.44 & 380.66 & 1.86 & 388.78 & 1.32 & 397.58 & 0.92 \\
\hline 8 & 392.79 & 4.81 & 401.05 & 3.68 & 408.79 & 2.77 & 416.04 & 2.10 & 423.03 & 1.65 & 431.50 & 1.31 & 442.12 & 1.06 \\
\hline 9 & 424.64 & 3.58 & 431.67 & 3.04 & 439.23 & 2.70 & 447.61 & 2.41 & 456.84 & 2.10 & 468.89 & 1.73 & 483.91 & 1.35 \\
\hline 10 & 456.90 & 4.14 & 465.85 & 3.61 & 475.36 & 3.08 & 485.36 & 2.58 & 495.77 & 2.14 & 508.55 & 1.65 & 523.18 & 1.17 \\
\hline 11 & 492.46 & 4.54 & 503.00 & 3.93 & 514.11 & 3.31 & 525.56 & 2.71 & 536.96 & 2.11 & 549.83 & 1.46 & 563.35 & 1.01 \\
\hline 12 & 532.52 & 4.74 & 544.15 & 3.93 & 555.70 & 3.08 & 566.51 & 2.26 & 576.14 & 1.60 & 586.75 & 1.19 & 600.74 & 1.10 \\
\hline 13 & 575.36 & 5.07 & 587.85 & 3.70 & 598.60 & 2.51 & 607.87 & 1.83 & 617.27 & 1.61 & 630.47 & 1.46 & 648.41 & 1.26 \\
\hline 14 & 605.50 & 4.33 & 616.96 & 3.35 & 628.59 & 2.90 & 641.70 & 2.65 & 656.35 & 2.31 & 674.87 & 1.80 & 696.15 & 1.32 \\
\hline 15 & 640.04 & 3.14 & 649.90 & 2.97 & 661.76 & 2.89 & 675.45 & 2.59 & 689.99 & 2.13 & 706.97 & 1.51 & 726.02 & 1.22 \\
\hline 16 & 678.49 & 4.30 & 692.81 & 3.95 & 708.19 & 3.31 & 723.29 & 2.49 & 736.56 & 1.69 & 749.71 & 1.10 & 767.53 & 1.24 \\
\hline 17 & 716.50 & 5.11 & 733.51 & 4.26 & 750.04 & 3.20 & 764.58 & 2.19 & 777.50 & 1.67 & 794.51 & 1.50 & 818.67 & 1.45 \\
\hline 18 & 755.05 & 5.10 & 771.67 & 3.75 & 785.91 & 2.55 & 799.06 & 2.11 & 814.39 & 2.04 & 835.69 & 1.71 & 862.65 & 1.48 \\
\hline 19 & 788.57 & 4.54 & 803.40 & 3.20 & 817.64 & 2.75 & 834.31 & 2.62 & 853.05 & 2.25 & 875.60 & 1.63 & 901.48 & 1.42 \\
\hline 20 & 825.12 & 3.06 & 837.40 & 2.94 & 853.36 & 3.07 & 872.03 & 2.72 & 890.97 & 2.08 & 911.30 & 1.37 & 937.25 & 1.49 \\
\hline 21 & 865.39 & 3.91 & 882.86 & 3.86 & 902.12 & 3.23 & 920.33 & 2.29 & 935.32 & 1.49 & 952.37 & 1.28 & 980.51 & 1.56 \\
\hline 22 & 901.73 & 5.28 & 924.05 & 4.45 & 945.61 & 3.27 & 963.81 & 2.14 & 980.44 & 1.78 & 1003.48 & 1.57 & 1035.34 & 1.58 \\
\hline 23 & 934.95 & 5.08 & 956.02 & 3.91 & 974.90 & 2.78 & 993.43 & 2.45 & 1015.47 & 2.31 & 1044.50 & 1.82 & 1079.78 & 1.60 \\
\hline 24 & 977.94 & 4.74 & 996.39 & 3.03 & 1012.01 & 2.40 & 1030.75 & 2.45 & 1052.67 & 2.14 & 1078.27 & 1.47 & 1110.15 & 1.59 \\
\hline
\end{tabular}

Note - All $\dot{P} / P$ values are in units of $10^{-16} \mathrm{~s}^{-1}$.

Table 5. Periods and relative rates of period change $(\ell=1$ modes $)$ for selected models with $M_{*}=0.65 M_{\odot}$ and $M_{\mathrm{He}}=9 \times 10^{-4} M_{*}$.

\begin{tabular}{|c|c|c|c|c|c|c|c|c|c|c|c|c|c|c|}
\hline \multirow[b]{2}{*}{$k$} & \multicolumn{2}{|c|}{$T_{\text {eff }}=28041 \mathrm{~K}$} & \multicolumn{2}{|c|}{$27090 \mathrm{~K}$} & \multicolumn{2}{|c|}{$26020 \mathrm{~K}$} & \multicolumn{2}{|c|}{$25024 \mathrm{~K}$} & \multicolumn{2}{|c|}{$23943 \mathrm{~K}$} & \multicolumn{2}{|c|}{$22945 \mathrm{~K}$} & \multicolumn{2}{|c|}{$22039 \mathrm{~K}$} \\
\hline & $P[\mathrm{~s}]$ & $\dot{P} / P$ & $P[\mathrm{~s}]$ & $\dot{P} / P$ & $P[\mathrm{~s}]$ & $\dot{P} / P$ & $P[\mathrm{~s}]$ & $\dot{P} / P$ & $P[\mathrm{~s}]$ & $\dot{P} / P$ & $P[\mathrm{~s}]$ & $\dot{P} / P$ & $P[\mathrm{~s}]$ & $\dot{P} / P$ \\
\hline 1 & 126.88 & 5.11 & 130.38 & 4.06 & 134.59 & 3.05 & 138.82 & 2.29 & 143.81 & 1.69 & 148.90 & 1.31 & 153.99 & 1.05 \\
\hline 2 & 153.06 & 3.74 & 156.19 & 3.06 & 160.05 & 2.38 & 164.01 & 1.83 & 168.78 & 1.39 & 173.71 & 1.09 & 178.62 & 0.87 \\
\hline 3 & 193.63 & 4.61 & 198.47 & 3.69 & 204.31 & 2.77 & 210.15 & 2.08 & 216.87 & 1.48 & 223.37 & 1.08 & 229.48 & 0.83 \\
\hline 4 & 227.51 & 3.60 & 231.93 & 2.89 & 237.25 & 2.17 & 242.58 & 1.65 & 248.81 & 1.22 & 255.24 & 0.98 & 261.98 & 0.84 \\
\hline 5 & 262.04 & 4.58 & 268.53 & 3.67 & 276.43 & 2.78 & 284.42 & 2.11 & 293.84 & 1.56 & 303.36 & 1.19 & 312.62 & 0.92 \\
\hline 6 & 300.74 & 3.87 & 306.90 & 3.00 & 314.08 & 2.17 & 321.07 & 1.62 & 329.02 & 1.15 & 336.68 & 0.85 & 343.89 & 0.66 \\
\hline 7 & 332.30 & 3.48 & 338.56 & 2.81 & 346.21 & 2.16 & 353.90 & 1.62 & 362.70 & 1.16 & 371.30 & 0.88 & 379.95 & 0.74 \\
\hline 8 & 377.94 & 3.54 & 384.60 & 2.47 & 391.56 & 1.63 & 398.06 & 1.23 & 406.14 & 1.02 & 415.55 & 0.92 & 425.99 & 0.80 \\
\hline 9 & 401.62 & 2.99 & 408.76 & 2.81 & 418.85 & 2.48 & 430.26 & 2.06 & 444.54 & 1.58 & 458.90 & 1.16 & 471.83 & 0.82 \\
\hline 10 & 438.27 & 3.84 & 447.38 & 3.09 & 458.37 & 2.30 & 469.24 & 1.71 & 481.02 & 1.12 & 491.21 & 0.75 & 501.22 & 0.69 \\
\hline 11 & 471.93 & 3.67 & 481.49 & 3.05 & 493.22 & 2.31 & 504.45 & 1.60 & 516.34 & 1.08 & 528.34 & 0.90 & 541.75 & 0.84 \\
\hline 12 & 508.90 & 4.11 & 519.69 & 3.02 & 530.98 & 1.89 & 540.66 & 1.32 & 552.67 & 1.14 & 567.07 & 1.01 & 582.65 & 0.88 \\
\hline 13 & 548.92 & 2.53 & 555.93 & 1.88 & 565.15 & 1.75 & 577.06 & 1.69 & 593.43 & 1.36 & 609.46 & 0.94 & 623.80 & 0.76 \\
\hline 14 & 578.30 & 3.71 & 590.30 & 3.15 & 605.61 & 2.49 & 621.19 & 1.85 & 637.61 & 1.14 & 651.14 & 0.77 & 666.40 & 0.86 \\
\hline 15 & 607.87 & 3.18 & 619.20 & 2.90 & 634.25 & 2.36 & 649.46 & 1.72 & 666.45 & 1.24 & 684.86 & 1.08 & 705.77 & 1.01 \\
\hline 16 & 648.21 & 4.04 & 662.06 & 3.09 & 676.56 & 1.85 & 687.85 & 1.16 & 701.46 & 1.05 & 718.58 & 0.96 & 738.44 & 0.96 \\
\hline 17 & 689.47 & 3.71 & 701.93 & 2.49 & 715.01 & 1.79 & 729.95 & 1.67 & 750.04 & 1.30 & 768.60 & 0.84 & 787.10 & 0.92 \\
\hline 18 & 720.75 & 3.26 & 733.19 & 2.61 & 749.99 & 2.33 & 769.03 & 1.89 & 790.64 & 1.24 & 809.79 & 0.90 & 832.91 & 1.06 \\
\hline 19 & 752.05 & 2.99 & 765.37 & 2.82 & 784.18 & 2.43 & 803.57 & 1.75 & 823.80 & 1.15 & 845.00 & 1.03 & 871.16 & 1.09 \\
\hline 20 & 783.56 & 3.72 & 800.55 & 3.31 & 821.32 & 2.36 & 839.23 & 1.47 & 859.00 & 1.21 & 882.52 & 1.05 & 909.66 & 1.10 \\
\hline 21 & 825.67 & 3.79 & 841.90 & 2.79 & 858.29 & 1.68 & 873.96 & 1.48 & 897.34 & 1.35 & 921.88 & 0.97 & 948.35 & 1.08 \\
\hline 22 & 867.99 & 3.87 & 883.99 & 2.49 & 900.69 & 1.87 & 920.13 & 1.68 & 943.76 & 1.14 & 963.98 & 0.79 & 991.11 & 1.14 \\
\hline 23 & 896.36 & 3.53 & 913.02 & 2.83 & 936.08 & 2.56 & 961.43 & 1.96 & 987.51 & 1.15 & 1011.52 & 0.96 & 1042.90 & 1.18 \\
\hline 24 & 927.94 & 2.72 & 943.81 & 2.82 & 967.15 & 2.42 & 990.17 & 1.64 & 1015.52 & 1.30 & 1046.29 & 1.18 & 1081.47 & 1.16 \\
\hline 25 & 968.77 & 3.58 & 988.78 & 3.13 & 1011.86 & 2.00 & 1030.38 & 1.30 & 1054.70 & 1.24 & 1082.21 & 0.94 & 1114.22 & 1.17 \\
\hline
\end{tabular}

Note - All $\dot{P} / P$ values are in units of $10^{-16} \mathrm{~s}^{-1}$. 
Table 6. Periods and relative rates of period change $(\ell=1$ modes $)$ for selected models with $M_{*}=0.70 M_{\odot}$ and $M_{\mathrm{He}}=9 \times 10^{-4} M_{*}$.

\begin{tabular}{|c|c|c|c|c|c|c|c|c|c|c|c|c|c|c|}
\hline \multirow[b]{2}{*}{$k$} & \multicolumn{2}{|c|}{$T_{\text {eff }}=28075 \mathrm{~K}$} & \multicolumn{2}{|c|}{$269997 \mathrm{~K}$} & \multicolumn{2}{|c|}{$25992 \mathrm{~K}$} & \multicolumn{2}{|c|}{$25055 \mathrm{~K}$} & \multicolumn{2}{|c|}{$24066 \mathrm{~K}$} & \multicolumn{2}{|c|}{$23033 \mathrm{~K}$} & \multicolumn{2}{|c|}{$222077 \mathrm{~K}$} \\
\hline & $P[\mathrm{~s}]$ & $\dot{P} / P$ & $P[\mathrm{~s}]$ & $\dot{P} / P$ & $P[\mathrm{~s}]$ & $\dot{P} / P$ & $P[\mathrm{~s}]$ & $\dot{P} / P$ & $P[\mathrm{~s}]$ & $\dot{P} / P$ & $P[\mathrm{~s}]$ & $\dot{P} / P$ & $P[\mathrm{~s}]$ & $\dot{P} / P$ \\
\hline 1 & 125.06 & 3.69 & 128.96 & 2.73 & 132.89 & 2.04 & 136.88 & 1.59 & 141.50 & 1.25 & 146.84 & 1.00 & 152.29 & 0.83 \\
\hline 2 & 147.72 & 2.93 & 151.42 & 2.25 & 155.22 & 1.69 & 159.10 & 1.33 & 163.60 & 1.04 & 168.71 & 0.82 & 173.82 & 0.67 \\
\hline 3 & 189.01 & 3.36 & 194.33 & 2.44 & 199.56 & 1.77 & 204.62 & 1.31 & 210.11 & 0.97 & 216.09 & 0.75 & 222.06 & 0.63 \\
\hline 4 & 217.33 & 2.67 & 222.22 & 1.98 & 227.15 & 1.49 & 232.17 & 1.19 & 238.21 & 0.99 & 245.69 & 0.86 & 253.91 & 0.77 \\
\hline 5 & 254.45 & 3.38 & 261.71 & 2.49 & 269.05 & 1.88 & 276.45 & 1.45 & 284.90 & 1.12 & 294.10 & 0.82 & 302.39 & 0.59 \\
\hline 6 & 288.43 & 2.60 & 294.68 & 1.89 & 300.90 & 1.41 & 306.98 & 1.05 & 313.58 & 0.78 & 320.83 & 0.62 & 328.75 & 0.60 \\
\hline 7 & 318.29 & 2.81 & 325.80 & 2.06 & 333.05 & 1.46 & 339.93 & 1.08 & 347.65 & 0.85 & 356.89 & 0.73 & 366.92 & 0.64 \\
\hline 8 & 359.95 & 1.90 & 365.57 & 1.38 & 371.61 & 1.18 & 378.66 & 1.08 & 388.00 & 0.95 & 399.26 & 0.77 & 410.14 & 0.59 \\
\hline 9 & 384.26 & 3.02 & 394.63 & 2.44 & 405.74 & 1.91 & 417.08 & 1.46 & 429.36 & 1.03 & 441.26 & 0.67 & 451.90 & 0.55 \\
\hline 10 & 421.26 & 2.73 & 430.87 & 1.98 & 440.02 & 1.37 & 448.11 & 0.91 & 456.43 & 0.70 & 467.42 & 0.72 & 481.62 & 0.74 \\
\hline 11 & 452.58 & 2.95 & 463.50 & 2.06 & 473.38 & 1.36 & 482.64 & 1.05 & 494.02 & 0.91 & 507.86 & 0.74 & 521.77 & 0.62 \\
\hline 12 & 489.07 & 2.35 & 497.76 & 1.49 & 506.56 & 1.28 & 517.18 & 1.19 & 530.81 & 0.99 & 546.17 & 0.75 & 561.51 & 0.67 \\
\hline 13 & 518.80 & 2.08 & 529.51 & 2.01 & 542.42 & 1.69 & 555.59 & 1.24 & 568.74 & 0.81 & 581.56 & 0.60 & 596.47 & 0.66 \\
\hline 14 & 555.63 & 2.93 & 569.52 & 2.19 & 582.78 & 1.48 & 594.12 & 0.96 & 606.27 & 0.80 & 622.33 & 0.75 & 641.22 & 0.75 \\
\hline 15 & 581.77 & 2.87 & 595.88 & 2.11 & 609.22 & 1.46 & 622.58 & 1.21 & 639.63 & 1.05 & 659.93 & 0.83 & 681.23 & 0.78 \\
\hline 16 & 622.35 & 2.44 & 633.07 & 1.35 & 642.99 & 1.14 & 655.41 & 1.12 & 671.50 & 0.91 & 689.33 & 0.70 & 709.76 & 0.77 \\
\hline 17 & 657.81 & 2.18 & 671.06 & 1.94 & 686.81 & 1.62 & 702.30 & 1.12 & 716.70 & 0.69 & 731.67 & 0.60 & 752.84 & 0.80 \\
\hline 18 & 688.04 & 2.70 & 705.03 & 2.24 & 722.58 & 1.62 & 738.56 & 1.12 & 756.40 & 0.93 & 778.48 & 0.78 & 803.75 & 0.84 \\
\hline 19 & 719.30 & 2.87 & 737.04 & 2.14 & 753.25 & 1.39 & 768.50 & 1.12 & 788.46 & 1.01 & 812.59 & 0.81 & 840.19 & 0.87 \\
\hline 20 & 755.15 & 2.95 & 772.03 & 1.77 & 786.70 & 1.31 & 803.73 & 1.23 & 824.86 & 0.95 & 847.08 & 0.71 & 874.44 & 0.88 \\
\hline 21 & 789.76 & 2.30 & 804.40 & 1.70 & 821.99 & 1.59 & 841.50 & 1.23 & 861.54 & 0.83 & 883.89 & 0.75 & 913.96 & 0.90 \\
\hline 22 & 828.10 & 2.13 & 844.91 & 1.95 & 864.04 & 1.49 & 881.14 & 0.98 & 899.61 & 0.83 & 924.27 & 0.76 & 955.39 & 0.90 \\
\hline 23 & 857.68 & 2.94 & 880.47 & 2.37 & 902.55 & 1.57 & 921.73 & 1.11 & 944.67 & 0.94 & 970.05 & 0.69 & 1001.49 & 0.92 \\
\hline 24 & 888.14 & 2.89 & 909.34 & 1.99 & 927.99 & 1.36 & 948.99 & 1.31 & 976.68 & 1.08 & 1007.50 & 0.83 & 1044.05 & 0.94 \\
\hline 25 & 930.34 & 2.70 & 948.56 & 1.56 & 966.30 & 1.39 & 987.57 & 1.17 & 1009.91 & 0.78 & 1035.44 & 0.76 & 1072.25 & 0.94 \\
\hline 26 & 967.99 & 2.51 & 987.79 & 1.90 & 1010.83 & 1.60 & 1032.55 & 1.04 & 1054.27 & 0.82 & 1082.24 & 0.73 & 1119.22 & 0.96 \\
\hline 27 & 998.51 & 2.41 & 1021.85 & 2.23 & 1047.28 & 1.59 & 1069.80 & 1.12 & 1097.37 & 0.99 & 1128.35 & 0.72 & 1167.09 & 0.97 \\
\hline
\end{tabular}

Note - All $\dot{P} / P$ values are in units of $10^{-16} \mathrm{~s}^{-1}$.

Our $\ell=1$ values of $\dot{P} / P$ range from $\sim 4$ to $\sim 7 \times 10^{-16} \mathrm{~s}^{-1}$ at $T_{\text {eff }} \approx 30000 \mathrm{~K}$ and from $\sim 1.5$ to $\sim 3 \times 10^{-16} \mathrm{~s}^{-1}$ at $T_{\text {eff }} \approx$ $25000 \mathrm{~K}$, for periods in the interval $\approx 100-1000 \mathrm{~s}$ in the case of a representative $0.60-M_{\odot}$ model, irrespective of the helium content. Our $\dot{P} / P$ values are almost twice as large as the values quoted by Kawaler et al. (1986) for a DB model with $0.60 M_{\odot}$ and $M_{\mathrm{He}} \approx 3.3 \times 10^{-2} M_{*}$ at $T_{\mathrm{eff}} \approx 30000 \mathrm{~K}$. The $\ell=1,2$ values of $\dot{P}$ for our models are about 4 times greater than those of Bradley \& Winget (1991) (their Table 12). This is mainly due to the distinct input physics employed and in particular to the fact that these authors do not consider neutrino emission in their Tassoul et al. (1990)'s carbon-core DB white dwarf models. As compared with the values of Bradley et al. (1993) (their Table 6 , corresponding to a $0.6-M_{\odot}$ carbon-core white dwarf model), our $\ell=2$ values of $\dot{P}$ are about 1.55 times greater, depending on the effective temperature. Unfortunately, the lack of extensive tabulations of $\dot{P}$ in the Bradley et al. (1993)'s paper prevent us from making a comprehensive comparison between their results and ours. We note that our models make use of a much more updated description of the opacities, rates of neutrino emission and core chemical composition than that used in Bradley et al. (1993).

The $\dot{P} / P$ values in our models exhibits a number of general trends. Firstly, we find that $\dot{P} / P$ generally decreases with decreasing effective temperature, reflecting the diminishing effect of neutrino cooling and the increase in the cooling timescale due to the gradual decreasing of the thermal energy content. Second, trapped modes in the helium envelope have smaller $\dot{P} / P$ values since these modes are concentrated closer to the surface, where gravitational contraction is still appreciable. As stated in Sect. 1, gravitational contraction acts shortening the pulsation periods, causing trapped modes to have smaller $\dot{P}$ values. This trend is in agreement with previous studies on DB white dwarf pulsations (see, e.g., Bradley et al. 1993).

Next, we shall briefly examine the effect of changing the various structural parameters on the rate of period change of our models. Factors that affect $\dot{P} / P$ in white dwarf models include the core composition, the surface chemical stratification, the stellar mass and the neutrino emission. Here we restrict ourselves to examining the effects of the total stellar mass, the mass of the helium layer and the rate of neutrino emission.

Effect of the stellar mass: $\dot{P} / P$ in white dwarf stars is very sensitive mostly to the stellar mass. The effect of the stellar mass on the rate of period change has been explored by Kawaler et al. (1986) for DB white dwarfs, by Kawaler et al. (1985a) for DO white dwarfs, and by Bradley \& Winget (1991), Bradley et al. (1993) and Bradley (1996) for DA white dwarfs. Lower mass models exhibit larger $\dot{P} / P$ values through the range of effective temperature of pulsating DB white dwarf stars (Kawaler et al. 1986). This can be understood on the basis that, if we consider a fixed $T_{\text {eff }}$ value, lower mass white dwarfs have larger 
Table 7. Periods and relative rates of period change $(\ell=1$ modes $)$ for selected models with $M_{*}=0.75 M_{\odot}$ and $M_{\mathrm{He}}=8 \times 10^{-3} M_{*}$.

\begin{tabular}{|c|c|c|c|c|c|c|c|c|c|c|c|c|c|c|}
\hline & \multicolumn{2}{|c|}{$T_{\text {eff }}=27969 \mathrm{~K}$} & \multicolumn{2}{|c|}{$27011 \mathrm{~K}$} & \multicolumn{2}{|c|}{$26002 \mathrm{~K}$} & \multicolumn{2}{|c|}{$25070 \mathrm{~K}$} & \multicolumn{2}{|c|}{$23956 \mathrm{~K}$} & \multicolumn{2}{|c|}{$23025 \mathrm{~K}$} & \multicolumn{2}{|c|}{$22022 \mathrm{~K}$} \\
\hline$k$ & $P[\mathrm{~s}]$ & $\dot{P} / P$ & $P[\mathrm{~s}]$ & $\dot{P} / P$ & $P[\mathrm{~s}]$ & $\dot{P} / P$ & $P[\mathrm{~s}]$ & $\dot{P} / P$ & $P[\mathrm{~s}]$ & $\dot{P} / P$ & $P[\mathrm{~s}]$ & $\dot{P} / P$ & $P[\mathrm{~s}]$ & $\dot{P} / P$ \\
\hline 1 & 115.07 & 2.03 & 118.26 & 1.61 & 121.98 & 1.30 & 125.83 & 1.10 & 130.99 & 0.92 & 135.77 & 0.81 & 141.33 & 0.71 \\
\hline 2 & 147.18 & 1.88 & 150.91 & 1.46 & 155.16 & 1.14 & 159.35 & 0.92 & 164.61 & 0.72 & 169.11 & 0.59 & 173.95 & 0.49 \\
\hline 3 & 173.02 & 1.43 & 176.28 & 1.08 & 179.89 & 0.83 & 183.44 & 0.69 & 188.17 & 0.60 & 192.78 & 0.57 & 198.63 & 0.55 \\
\hline 4 & 213.57 & 1.72 & 218.49 & 1.33 & 224.12 & 1.06 & 229.91 & 0.90 & 237.66 & 0.76 & 244.68 & 0.65 & 252.41 & 0.53 \\
\hline 5 & 236.16 & 1.64 & 241.58 & 1.36 & 248.16 & 1.14 & 255.04 & 0.97 & 264.13 & 0.79 & 272.26 & 0.67 & 281.49 & 0.59 \\
\hline 6 & 277.38 & 1.66 & 283.33 & 1.19 & 289.30 & 0.80 & 294.32 & 0.57 & 300.19 & 0.45 & 305.85 & 0.45 & 313.36 & 0.46 \\
\hline 7 & 297.53 & 1.40 & 303.14 & 1.10 & 309.88 & 0.95 & 317.46 & 0.89 & 328.60 & 0.82 & 339.35 & 0.73 & 351.84 & 0.63 \\
\hline 8 & 336.46 & 1.03 & 341.60 & 0.95 & 348.47 & 0.87 & 355.90 & 0.74 & 365.24 & 0.56 & 372.78 & 0.44 & 380.97 & 0.40 \\
\hline 9 & 372.43 & 2.01 & 382.53 & 1.55 & 393.38 & 1.09 & 402.32 & 0.70 & 411.34 & 0.48 & 419.17 & 0.44 & 429.07 & 0.45 \\
\hline 10 & 396.26 & 1.41 & 403.35 & 0.99 & 410.92 & 0.79 & 419.88 & 0.85 & 435.03 & 0.87 & 450.46 & 0.79 & 468.69 & 0.70 \\
\hline 11 & 435.31 & 1.27 & 442.23 & 0.91 & 450.48 & 0.81 & 459.61 & 0.71 & 471.35 & 0.55 & 481.21 & 0.46 & 493.41 & 0.51 \\
\hline 12 & 457.41 & 1.47 & 467.69 & 1.40 & 480.84 & 1.17 & 494.06 & 0.93 & 509.82 & 0.68 & 522.69 & 0.55 & 538.12 & 0.58 \\
\hline 13 & 495.52 & 1.50 & 505.36 & 1.11 & 515.23 & 0.75 & 524.02 & 0.60 & 536.72 & 0.60 & 550.82 & 0.62 & 570.27 & 0.68 \\
\hline 14 & 528.02 & 1.73 & 539.38 & 1.17 & 550.72 & 0.86 & 562.24 & 0.74 & 577.67 & 0.61 & 591.10 & 0.51 & 608.65 & 0.63 \\
\hline 15 & 556.80 & 1.27 & 566.46 & 1.06 & 579.67 & 1.03 & 594.65 & 0.91 & 613.88 & 0.69 & 629.75 & 0.56 & 650.51 & 0.69 \\
\hline 16 & 583.05 & 1.41 & 595.15 & 1.26 & 609.90 & 1.02 & 624.25 & 0.79 & 642.26 & 0.66 & 660.27 & 0.66 & 685.76 & 0.76 \\
\hline 17 & 619.08 & 1.47 & 631.67 & 1.16 & 644.78 & 0.80 & 656.76 & 0.66 & 673.88 & 0.62 & 690.99 & 0.58 & 715.23 & 0.74 \\
\hline 18 & 659.60 & 1.62 & 672.23 & 1.00 & 684.72 & 0.78 & 698.55 & 0.73 & 717.55 & 0.60 & 733.91 & 0.51 & 759.14 & 0.79 \\
\hline 19 & 683.63 & 1.48 & 697.58 & 1.24 & 715.78 & 1.12 & 734.86 & 0.90 & 757.29 & 0.64 & 776.21 & 0.57 & 804.98 & 0.81 \\
\hline 20 & 715.69 & 1.25 & 729.03 & 1.16 & 746.06 & 0.96 & 762.71 & 0.77 & 785.46 & 0.72 & 808.91 & 0.69 & 841.61 & 0.82 \\
\hline 21 & 746.97 & 1.59 & 763.68 & 1.28 & 781.17 & 0.88 & 797.14 & 0.72 & 819.76 & 0.66 & 841.48 & 0.60 & 874.08 & 0.84 \\
\hline 22 & 782.59 & 1.56 & 797.99 & 1.07 & 814.10 & 0.86 & 832.45 & 0.82 & 857.35 & 0.65 & 878.77 & 0.56 & 912.26 & 0.85 \\
\hline 23 & 818.75 & 1.44 & 833.23 & 1.01 & 851.15 & 0.93 & 870.18 & 0.76 & 893.50 & 0.60 & 915.85 & 0.60 & 951.68 & 0.85 \\
\hline 24 & 846.51 & 1.55 & 865.32 & 1.35 & 888.22 & 1.06 & 908.75 & 0.75 & 933.41 & 0.62 & 956.87 & 0.57 & 994.35 & 0.89 \\
\hline 25 & 873.67 & 1.35 & 891.63 & 1.25 & 913.03 & 0.97 & 935.22 & 0.90 & 968.32 & 0.80 & 997.63 & 0.65 & 1038.55 & 0.90 \\
\hline 26 & 915.25 & 1.52 & 933.00 & 1.04 & 950.10 & 0.76 & 969.13 & 0.74 & 995.97 & 0.63 & 1022.32 & 0.65 & 1065.09 & 0.86 \\
\hline 27 & 952.28 & 1.64 & 971.17 & 1.09 & 992.43 & 0.95 & 1014.28 & 0.73 & 1039.24 & 0.55 & 1063.09 & 0.55 & 1105.62 & 0.92 \\
\hline 28 & 980.15 & 1.47 & 1000.22 & 1.27 & 1026.48 & 1.08 & 1051.18 & 0.80 & 1082.11 & 0.67 & 1109.78 & 0.57 & 1154.29 & 0.93 \\
\hline
\end{tabular}

Note - All $\dot{P} / P$ values are in units of $10^{-16} \mathrm{~s}^{-1}$.

luminosities and lower total heat capacity, and thus cool faster with a larger $\dot{P}$. This can be also understood in terms of the simple, yet accurate enough for our purposes, cooling model of Mestel (1952). Within the framework of the Mestel (1952) cooling law, Kawaler et al. (1986) have derived a relation between $\dot{P} / P$ and the stellar mass:

$\frac{\mathrm{d} \ln P}{\mathrm{~d} t}=2 \times 10^{-30} A\left(\frac{\mu}{\mu_{\mathrm{e}}^{2}}\right)^{0.286}\left(\frac{M}{M_{\odot}}\right)^{-1.190} T_{\mathrm{eff}}^{2.857}$,

where $\mu$ is the mean molecular weight, $\mu_{\mathrm{e}}$ the molecular weight per electron, and $A$ the atomic mass of the ions. In particular, it is convenient to note that from our numerical computations the magnitude of the period derivative varies by about a factor of 6-7 in the stellar mass interval $0.50-0.85 M_{\odot}$, as we show in Fig. 2 for DB models with a helium layer mass of $\approx 8 \times 10^{-3} M_{*}$. Each panel in the figure illustrates the situation at different effective temperatures covering the observed DBV instability strip. As we can see from the figure, the general theoretical expectations from Eq. (2) are borne out by our detailed numerical computations. In fact, the rate of period change for the less massive models is always larger than for the more massive ones.

Figure 2 further emphasizes the complex structure characterizing the $\dot{P} / P$-distribution: no matter the stellar mass is, the diagrams show abundant local maxima and minima. The mode with $k=14$ corresponding to the $0.50-M_{\odot}$ white dwarf model at $T_{\text {eff }} \approx 30000 \mathrm{~K}$ (pointed with the arrow in left panel of the figure) is striking. The very pronounced minima in the rate of period change of this mode is not the result of a numerical glitch; we have verified the reality of this by examining their oscillation kinetic energy and eigenfunctions, and we have found that this mode is strongly trapped in the helium-rich envelope. As we have already anticipated, trapped modes like this one must have a small $\dot{P} / P$ value, reflecting primarily the contraction of the outer layers, rather than carrying information about the processes linked to the cooling of the white dwarf (Bradley et al. 1993). We have verified that almost all the trapped modes in the helium-rich envelope of our models correspond to local minima in $\dot{P} / P$.

Effect of the helium layer mass: Now we examine the possible dependence of the $\dot{P} / P$ values on the thickness of the helium layer, holding the stellar mass fixed. We have considered the following values for the helium mass: $1 \times 10^{-4} M_{*}, 9 \times 10^{-4} M_{*}$, and $8 \times 10^{-3} M_{*}$. It is worth recalling that in the sequence of DB models with $M_{\mathrm{He}} \approx 1 \times 10^{-4} M_{*}$ the envelope is characterized by an initially double-layered configuration which evolves to a single-layered structure before the model reaches the red edge of the DBV instability strip (Althaus \& Córsico 2004). The effects of varying the mass of the helium layer, keeping the total mass constant, are illustrated in Fig. 3. This figure 
Table 8. Periods and relative rates of period change $(\ell=1$ modes $)$ for selected models with $M_{*}=0.75 M_{\odot}$ and $M_{\mathrm{He}}=9 \times 10^{-4} M_{*}$.

\begin{tabular}{|c|c|c|c|c|c|c|c|c|c|c|c|c|c|c|}
\hline & \multicolumn{2}{|c|}{$\overline{T_{\text {eff }}=28041 \mathrm{~K}}$} & \multicolumn{2}{|c|}{$27039 \mathrm{~K}$} & \multicolumn{2}{|c|}{$25990 \mathrm{~K}$} & \multicolumn{2}{|c|}{$25029 \mathrm{~K}$} & \multicolumn{2}{|c|}{$24001 \mathrm{~K}$} & \multicolumn{2}{|c|}{$23033 \mathrm{~K}$} & \multicolumn{2}{|c|}{$21997 \mathrm{~K}$} \\
\hline$k$ & $P[\mathrm{~s}]$ & $\dot{P} / P$ & $P[\mathrm{~s}]$ & $\dot{P} / P$ & $P[\mathrm{~s}]$ & $\dot{P} / P$ & $P[\mathrm{~s}]$ & $\dot{P} / P$ & $P[\mathrm{~s}]$ & $\dot{P} / P$ & $P[\mathrm{~s}]$ & $\dot{P} / P$ & $P[\mathrm{~s}]$ & $\dot{P} / P$ \\
\hline 1 & 123.03 & 2.40 & 126.67 & 1.81 & 130.85 & 1.40 & 135.08 & 1.15 & 140.02 & 0.94 & 145.06 & 0.79 & 150.74 & 0.65 \\
\hline 2 & 142.97 & 2.02 & 146.53 & 1.53 & 150.59 & 1.18 & 154.64 & 0.95 & 159.26 & 0.76 & 163.89 & 0.64 & 69.15 & 0.55 \\
\hline 3 & 183.88 & 2.04 & 188.41 & 1.48 & 193.25 & 1.07 & 197.86 & 0.84 & 203.16 & 0.70 & 208.78 & 0.63 & & 0.58 \\
\hline 4 & 207.81 & 1.77 & 212.37 & 1.37 & 217.80 & 1.12 & 223.67 & 0.99 & 231.05 & 0.87 & 239.05 & 0.77 & 248.49 & 0.66 \\
\hline 5 & 246.86 & 2.18 & 253.53 & 1.66 & 261.12 & 1.26 & 268.46 & 0.97 & 276.19 & 0.69 & 282.82 & 0.49 & 89.35 & 0.38 \\
\hline 6 & 275.46 & 1.67 & 281.04 & 1.23 & 287.05 & 0.89 & 292.70 & 0.70 & 299.52 & 0.64 & 307.70 & 0.65 & 318.76 & 0.64 \\
\hline 7 & 306 & 1.78 & 312 & 1.24 & 3 & 0.94 & 32 & 0.82 & & 0.72 & & 0.61 & & 0.50 \\
\hline 8 & 340.44 & 1.33 & 346.67 & 1.23 & 355.13 & 1.10 & 364.26 & 0.91 & 374.49 & 0.70 & 383.97 & 0.54 & 394.41 & 0.48 \\
\hline 9 & 371.00 & 2.22 & 381.23 & 1.69 & 392.44 & 1.20 & 402.31 & 0.83 & 412 & 0.61 & 422.03 & 0.55 & 434 & 0.55 \\
\hline 10 & 403.20 & 1.61 & 410.54 & 1.04 & 417.71 & 0.74 & 425.52 & 0.74 & 437.16 & 0.77 & 450.95 & 0.71 & 467.73 & 0.65 \\
\hline 11 & 434.69 & 1.68 & 443.23 & 1.20 & 453.24 & 1.00 & 463.96 & 0.85 & 476.07 & 0.65 & 487.31 & 0.50 & 500 & 0.55 \\
\hline 12 & 464.85 & 1.40 & 474.03 & 1.33 & 486.22 & 1.12 & 498.52 & 0.88 & 511 & 0.68 & 525.31 & 0.58 & 542 & 0.63 \\
\hline 13 & 496.77 & 2.00 & 508. & 1.4 & 521.24 & 0. & 531.50 & 0. & 543 & 0.62 & 557.39 & 0.60 & 576 & 0.68 \\
\hline 14 & 53 & 1.79 & 544. & 1. & 555.10 & 0.90 & 567.52 & 0.84 & 583 & 0.72 & 598.64 & 0.56 & 618 & 0.69 \\
\hline 15 & 557.91 & 1.73 & 569.60 & 1.32 & 584.14 & 1.14 & 599.77 & 0.95 & 617 & 0.75 & 635.09 & 0.63 & & 0.77 \\
\hline 16 & 590.63 & 1.24 & 601.20 & 1.22 & 615.44 & 1.03 & 629.50 & 0.79 & 645.04 & 0.65 & 662.22 & 0.63 & 687.30 & 0.76 \\
\hline 17 & 629.79 & 1.92 & 644.3 & 1.3 & 658.04 & 0.81 & 669.64 & 0.64 & 684.65 & 0.62 & 701.79 & 0.57 & 727.55 & 0.81 \\
\hline 18 & 660.68 & 1.96 & 675.90 & 1.3 & 692.33 & 1.0 & 710.02 & 0.9 & 729.99 & 0.6 & 747.86 & 0.53 & 775.34 & 0.86 \\
\hline 19 & 690.22 & 1.68 & 703.4 & 1.1 & 720.07 & 1.08 & 738.48 & 0.91 & 759.95 & 0.76 & 782.97 & 0.69 & 15.68 & 0.85 \\
\hline 20 & 722.13 & 1.45 & 736.73 & 1.36 & 755.60 & 1.09 & 773.17 & 0.78 & 792.29 & 0.66 & 813.65 & 0.63 & 46.69 & 0.87 \\
\hline 21 & 754.41 & 1.81 & 772.02 & 1.4 & 790.43 & 0. & 807.32 & 0. & 829.33 & 0.73 & 852.75 & 0.63 & 887.27 & 0.89 \\
\hline 22 & & & & 1. & 825.20 & 0. & & & & & & & & 0.90 \\
\hline 23 & 825.01 & 1.98 & 843.3 & 1.3 & 864.24 & 1. & 885.33 & 0. & 907.13 & 0.6 & 930.08 & 0.59 & 968.71 & 0.94 \\
\hline 24 & 850.86 & 1.48 & 867.65 & 1.35 & 890.98 & 1.18 & 914.75 & 0.93 & 942.67 & 0.80 & 971.92 & 0.68 & 1013.92 & 0.93 \\
\hline
\end{tabular}

Note - All $\dot{P} / P$ values are in units of $10^{-16} \mathrm{~s}^{-1}$.

shows that the $\dot{P} / P$ values corresponding to models with massive helium envelopes are slightly smaller than the case of thinner ones. This effect can be explained on the basis that our models with massive helium layers cool slightly more slowly as compared with models characterized by thinner helium envelopes. This is understood on the basis that models with massive (and also more transparent) helium envelope are characterized by lower central temperatures which in turn, implies that such models have initially an excess of internal energy to get rid of - see Tassoul et al. (1990). This leads to longer cooling age at this stage and thus smaller rate of period variations.

Effect of the neutrino emission: Finally, we shall examine the effect of the neutrino emission on the $\dot{P} / P$ values. We begin by examining Fig. 4 , in which we show the evolution of the rate of period change in terms of the effective temperature corresponding to a sequence of $0.60-M_{\odot}$ white dwarf models with a helium content of $M_{\mathrm{He}}=8 \times 10^{-3} M_{*}$. Continuous lines correspond to the case in which neutrino emission has been included in our DB models, and short-dashed lines correspond to the situation in which neutrino emission has been ignored ${ }^{5}$. At the hot edge of the DB instability strip, the values of $\dot{P} / P$ considering neutrino emission are greater by a factor of about 5 as compared with calculations in which neutrino emission has not been taken into account. At $T_{\text {eff }} \approx 25000 \mathrm{~K}$ the factor is of

\footnotetext{
${ }^{5}$ It is worth mentioning that we have turned off neutrino emission at a high effective temperature $(\approx 60000 \mathrm{~K})$. As a result, the model has adequately readjusted its thermal structure long before the DBV regime is reached.
}

about 2.5 . In Fig. 5 we illustrate $\dot{P} / P$ versus periods at three effective temperatures, for the case in which neutrino losses have been considered (continuous lines) and for the situation in which neutrino emission has been ignored (dashed lines). Clearly, the $\dot{P} / P$ values are very sensitive to neutrino emission, particularly at the high effective temperatures characterizing the blue edge of the DBV instability strip. Thus, we essentially recover the results reported by Winget et al. (2004). We conclude, in agreement with Winget et al. (2004)'s claims, that at high effective temperatures within the DB instability strip the eventual detection of the rate of period change in DBV white dwarfs could allow the astronomers to constrain the production rates of plasmon neutrino emission.

\section{Summary}

The rate of period change is the most exciting observable quantity in pulsating white dwarf stars because it potentially provides a direct measure of the rate of cooling of white dwarfs, giving astronomers the possibility of inferring the age of the galactic disk in the solar neighborhood (Winget et al. 1987). Also, measurement of $\dot{P}$ in white dwarfs offers an unique opportunity to place interesting constraints on neutrino physics (O'Brien \& Kawaler 2000; Winget et al. 2004) and to test any additional sink of energy (Isern et al. 1992; Córsico et al. 2001a). In addition, the small magnitude of $\dot{P}$ characterizing pulsating white dwarfs implicates that its detection would also impose strong constraints on the presence of other mechanisms acting on shorter time scales and that can modify the 
Table 9. Periods and relative rates of period change $(\ell=1$ modes $)$ for selected models with $M_{*}=0.85 M_{\odot}$ and $M_{\mathrm{He}}=8 \times 10^{-3} M_{*}$.

\begin{tabular}{|c|c|c|c|c|c|c|c|c|c|c|c|c|c|c|}
\hline & \multicolumn{2}{|c|}{$T_{\mathrm{eff}}=27968 \mathrm{~K}$} & \multicolumn{2}{|c|}{$27040 \mathrm{~K}$} & \multicolumn{2}{|c|}{$26010 \mathrm{~K}$} & \multicolumn{2}{|c|}{$25003 \mathrm{~K}$} & \multicolumn{2}{|c|}{$24014 \mathrm{~K}$} & \multicolumn{2}{|c|}{$23038 \mathrm{~K}$} & \multicolumn{2}{|c|}{$21960 \mathrm{~K}$} \\
\hline$k$ & $P[\mathrm{~s}]$ & $\dot{P} / P$ & $P[\mathrm{~s}]$ & $\dot{P} / P$ & $P[\mathrm{~s}]$ & $\dot{P} / P$ & $P[\mathrm{~s}]$ & $\dot{P} / P$ & $P[\mathrm{~s}]$ & $\dot{P} / P$ & $P[\mathrm{~s}]$ & $\dot{P} / P$ & $P[\mathrm{~s}]$ & $\dot{P} / P$ \\
\hline 1 & 110.97 & 1.11 & 114.43 & 0.99 & 118.52 & 0.87 & 122.70 & 0.77 & 126.95 & 0.68 & 131.23 & 0.60 & 136.07 & 0.53 \\
\hline 2 & 138.36 & 0.89 & 141.68 & 0.74 & 145.34 & 0.61 & 148.88 & 0.53 & 152.39 & 0.47 & 155.97 & 0.43 & 160.18 & 0.40 \\
\hline 3 & 157.44 & 0.69 & 160.61 & 0.66 & 164.70 & 0.65 & 169.33 & 0.64 & 174.42 & 0.61 & 179.87 & 0.57 & 186.28 & 0.52 \\
\hline 4 & 197.77 & 0.97 & 203.09 & 0.84 & 209.16 & 0.71 & 215.00 & 0.59 & 220.48 & 0.49 & 225.60 & 0.41 & 231.07 & 0.34 \\
\hline 5 & 218.20 & 0.96 & 223.92 & 0.82 & 230.50 & 0.72 & 237.27 & 0.65 & 244.43 & 0.61 & 252.06 & 0.58 & 261.21 & 0.53 \\
\hline 6 & 250.83 & 0.53 & 254.69 & 0.51 & 259.78 & 0.52 & 265.70 & 0.53 & 272.30 & 0.51 & 279.35 & 0.48 & 287.60 & 0.43 \\
\hline 7 & 272.95 & 0.97 & 280.58 & 0.89 & 289.71 & 0.79 & 298.95 & 0.69 & 308.06 & 0.59 & 316.93 & 0.51 & 326.78 & 0.45 \\
\hline 8 & 305.06 & 0.72 & 310.74 & 0.56 & 316.74 & 0.46 & 322.75 & 0.43 & 329.28 & 0.42 & 336.65 & 0.43 & 346.15 & 0.43 \\
\hline 9 & 343.00 & 0.72 & 349.43 & 0.58 & 356.75 & 0.52 & 364.30 & 0.47 & 371.99 & 0.42 & 379.78 & 0.38 & 389.45 & 0.40 \\
\hline 10 & 356.75 & 0.90 & 366.63 & 0.92 & 379.39 & 0.87 & 392.95 & 0.78 & 406.73 & 0.68 & 419.94 & 0.56 & 434.29 & 0.50 \\
\hline 11 & 394.17 & 0.67 & 401.17 & 0.55 & 408.87 & 0.46 & 416.67 & 0.43 & 425.18 & 0.43 & 435.31 & 0.48 & 450.50 & 0.56 \\
\hline 12 & 425.26 & 0.91 & 435.47 & 0.73 & 446.45 & 0.60 & 457.01 & 0.51 & 467.35 & 0.45 & 477.68 & 0.41 & 492.31 & 0.51 \\
\hline 13 & 446.89 & 0.67 & 456.16 & 0.71 & 468.92 & 0.72 & 483.14 & 0.68 & 497.84 & 0.60 & 512.53 & 0.53 & 531.22 & 0.57 \\
\hline 14 & 481.73 & 0.78 & 491.86 & 0.65 & 502.81 & 0.53 & 513.53 & 0.47 & 524.89 & 0.46 & 537.71 & 0.48 & 557.25 & 0.59 \\
\hline 15 & 507.76 & 0.90 & 520.29 & 0.77 & 534.50 & 0.66 & 548.43 & 0.56 & 562.03 & 0.49 & 575.61 & 0.45 & 596.25 & 0.59 \\
\hline 16 & 534.22 & 0.72 & 545.23 & 0.68 & 559.60 & 0.68 & 575.86 & 0.66 & 593.21 & 0.60 & 610.84 & 0.54 & 635.62 & 0.65 \\
\hline 17 & 563.02 & 0.75 & 575.07 & 0.69 & 589.20 & 0.60 & 603.37 & 0.53 & 618.17 & 0.50 & 634.77 & 0.53 & 660.20 & 0.63 \\
\hline 18 & 599.01 & 0.78 & 611.48 & 0.64 & 625.34 & 0.55 & 639.44 & 0.50 & 654.11 & 0.47 & 669.82 & 0.47 & 696.61 & 0.67 \\
\hline 19 & 625.97 & 0.92 & 641.62 & 0.78 & 659.54 & 0.68 & 677.42 & 0.59 & 694.88 & 0.50 & 712.38 & 0.48 & 742.25 & 0.70 \\
\hline 20 & 651.11 & 0.71 & 665.00 & 0.72 & 683.43 & 0.70 & 703.55 & 0.66 & 724.56 & 0.60 & 746.35 & 0.56 & 778.67 & 0.69 \\
\hline 21 & 684.35 & 0.78 & 699.15 & 0.68 & 716.09 & 0.59 & 733.41 & 0.54 & 752.23 & 0.53 & 773.39 & 0.55 & 807.38 & 0.71 \\
\hline 22 & 714.41 & 0.84 & 730.59 & 0.70 & 748.78 & 0.61 & 767.61 & 0.56 & 787.18 & 0.51 & 807.93 & 0.52 & 843.11 & 0.71 \\
\hline 23 & 744.68 & 0.75 & 760.36 & 0.68 & 780.00 & 0.65 & 800.68 & 0.58 & 821.42 & 0.51 & 843.04 & 0.51 & 879.78 & 0.71 \\
\hline 24 & 775.02 & 0.82 & 792.77 & 0.73 & 813.23 & 0.62 & 833.46 & 0.55 & 854.48 & 0.51 & 877.27 & 0.52 & 916.02 & 0.72 \\
\hline 25 & 799.37 & 0.88 & 819.63 & 0.81 & 843.63 & 0.71 & 867.58 & 0.61 & 890.98 & 0.53 & 914.71 & 0.52 & 956.48 & 0.75 \\
\hline 26 & 831.80 & 0.73 & 848.10 & 0.62 & 868.29 & 0.62 & 892.12 & 0.64 & 919.11 & 0.62 & 948.18 & 0.60 & 991.63 & 0.71 \\
\hline 27 & 869.63 & 0.71 & 886.65 & 0.63 & 906.96 & 0.56 & 927.36 & 0.50 & 948.68 & 0.47 & 973.21 & 0.53 & 1017.88 & 0.75 \\
\hline 28 & 896.10 & 0.93 & 919.68 & 0.82 & 945.10 & 0.63 & 968.17 & 0.52 & 990.78 & 0.47 & 1014.94 & 0.49 & 1062.17 & 0.77 \\
\hline 29 & 922.59 & 0.80 & 943.71 & 0.74 & 970.29 & 0.72 & 999.84 & 0.67 & 1028.90 & 0.55 & 1056.64 & 0.52 & 1105.71 & 0.76 \\
\hline 30 & 955.46 & 0.80 & 975.90 & 0.67 & 1000.25 & 0.63 & 1026.53 & 0.59 & 1055.53 & 0.59 & 1088.05 & 0.59 & 1139.19 & 0.74 \\
\hline 31 & 986.08 & 0.75 & 1007.39 & 0.71 & 1033.53 & 0.63 & 1060.00 & 0.56 & 1087.32 & 0.52 & 1117.51 & 0.56 & 1170.46 & 0.76 \\
\hline
\end{tabular}

Note - All $\dot{P} / P$ values are in units of $10^{-16} \mathrm{~s}^{-1}$.

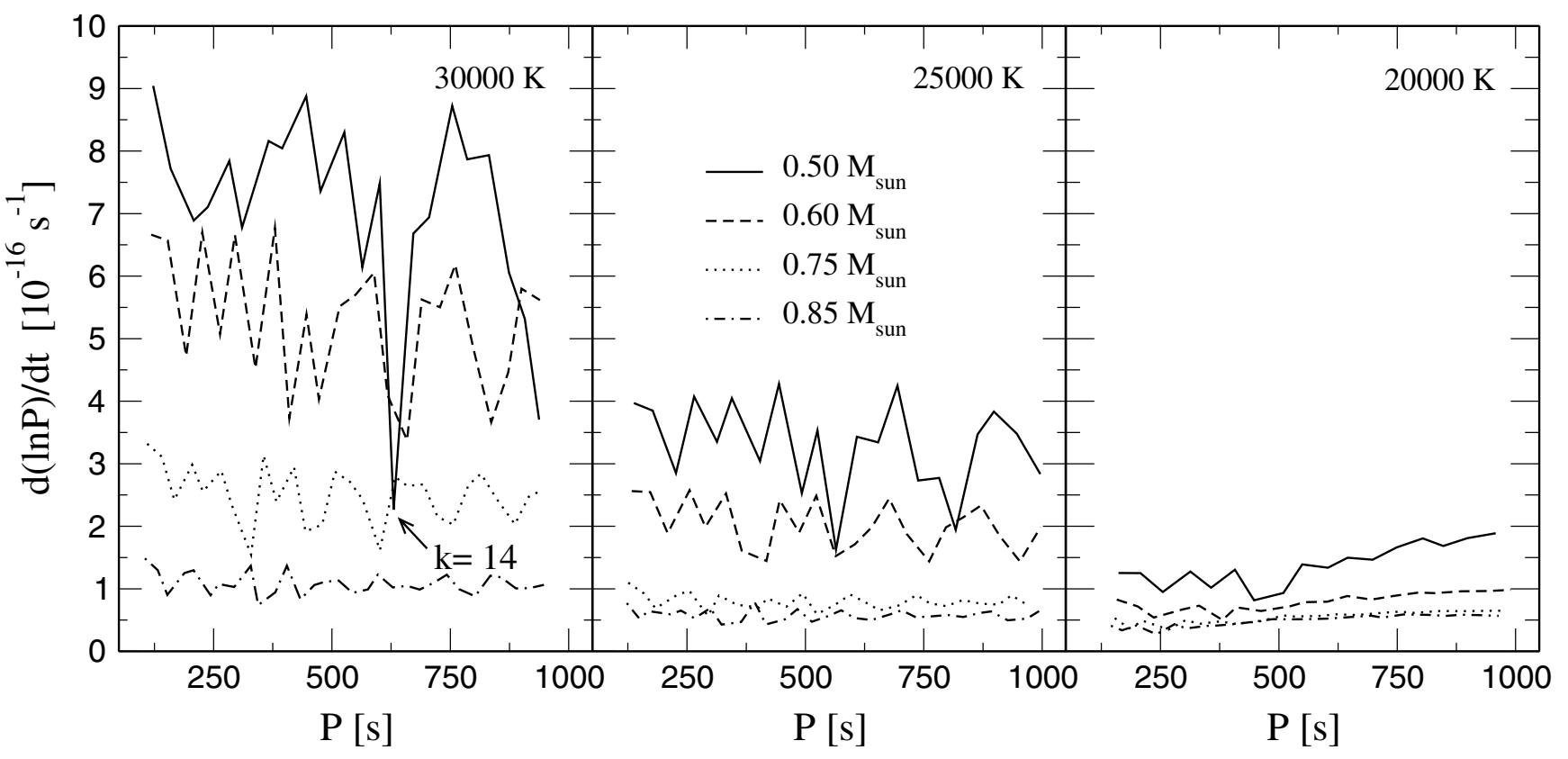

Fig. 2. The $\dot{P} / P$ values versus the period, corresponding to DB white dwarf models with stellar masses from 0.50 to $0.85 M_{\odot}$ (indicated with different line styles) and a helium content of $M_{\mathrm{He}}=8 \times 10^{-3} M_{*}$. Left, centre and right panels display the results for effective temperatures of 30000,25000 , and $20000 \mathrm{~K}$, respectively. Note: the strong minimum in $\dot{P} / P$ pointed with the arrow is not numerically inflicted (see text). 


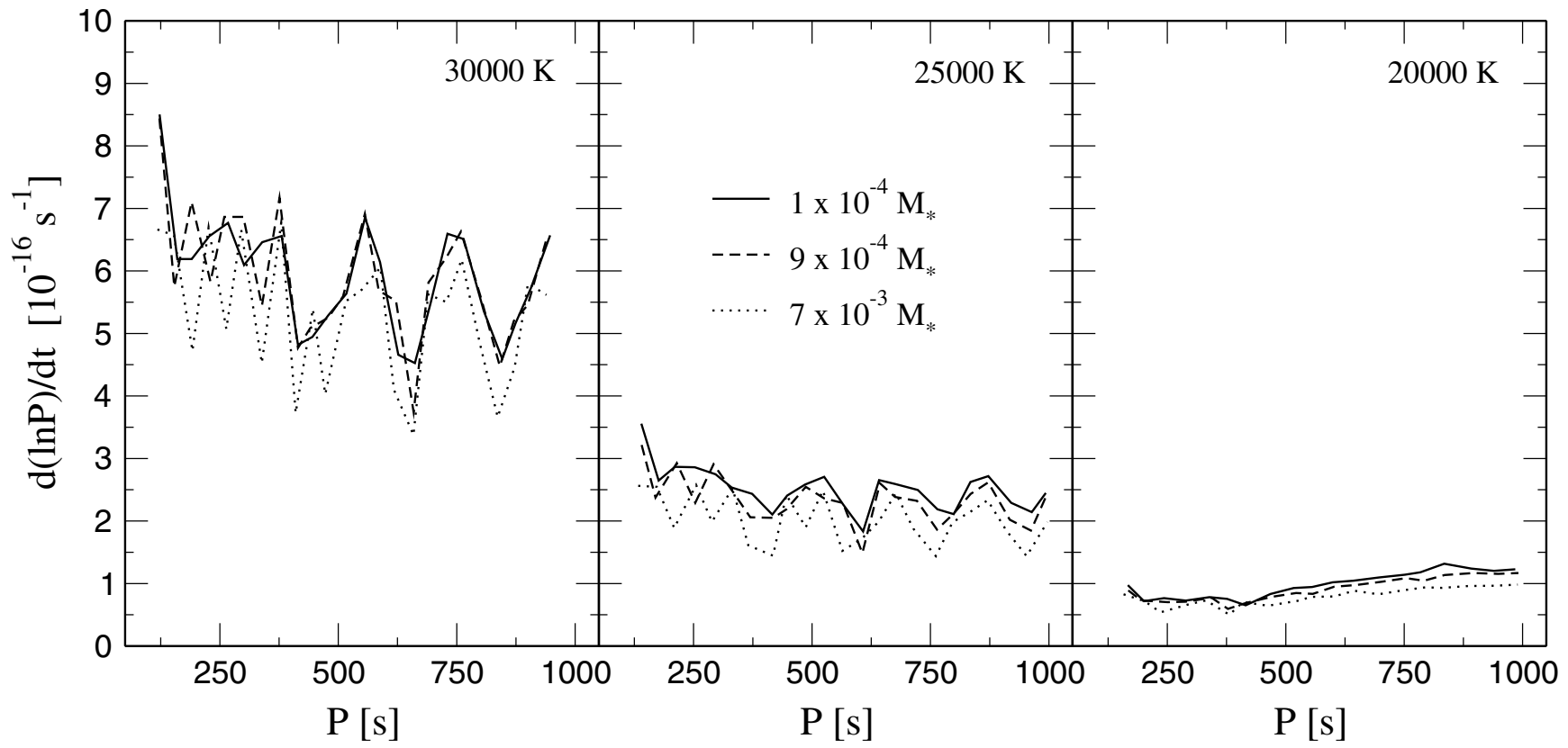

Fig. 3. The $\dot{P} / P$ values versus the period, corresponding to DB white dwarf models with a stellar mass of $0.60 M_{\odot}$ and a content of helium of $1 \times 10^{-4} M_{*}$ (solid lines), $9 \times 10^{-4} M_{*}$ (dashed lines), and $8 \times 10^{-3} M_{*}$ (dotted lines). Left, centre and right panels display the results for effective temperatures of 30000,25000 , and $20000 \mathrm{~K}$, respectively. $\dot{P} / P$ values corresponding to models with thick helium envelopes are only marginally smaller as compared with the case of thin helium envelopes.

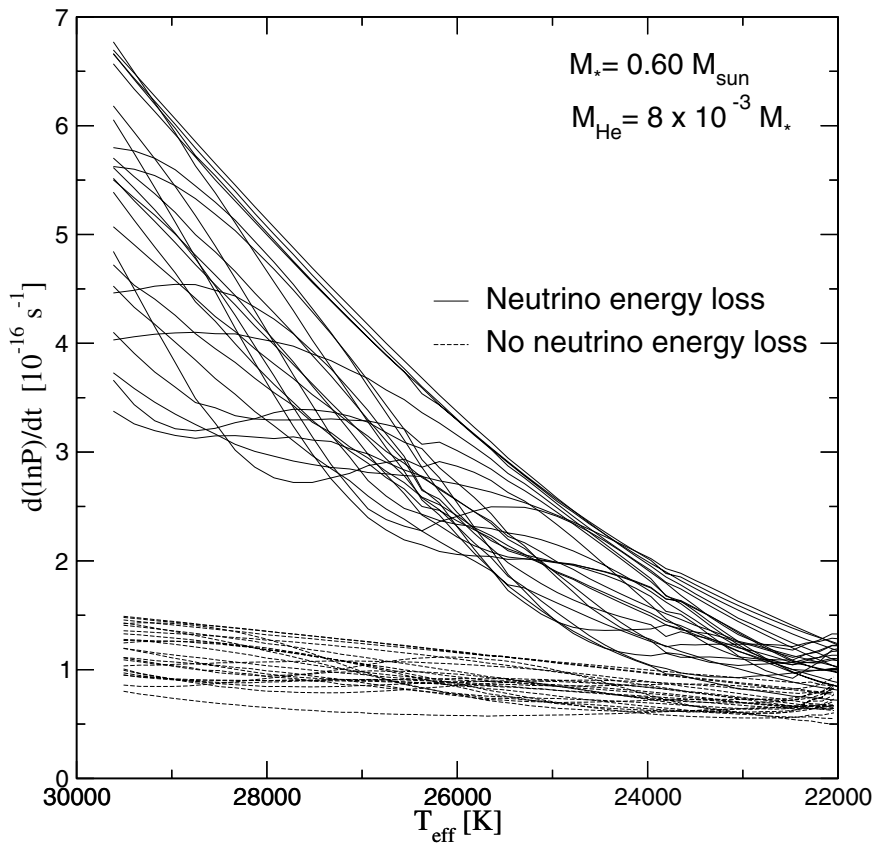

Fig. 4. The evolution of the relative rate of period change including (continuous lines) and ignoring (short-dashed lines) the effect of neutrino, in terms of the effective temperature, corresponding to a sequence of $0.60-M_{\odot}$ white dwarf models $\left(M_{\mathrm{He}}=8 \times 10^{-3} M_{*}\right)$.

pulsation periods, such as stellar rotation (Kawaler et al. 1985b) and binary orbital motion (Kepler et al. 1991).

Rates of period change have been detected for two members of the ZZ Ceti variable class (G117-B15A and R548) and only for a single DOV white dwarf (PG 1159-035). In the case of $\mathrm{ZZ}$ Ceti stars, the observed values for the rate of period change are in good agreement with the theoretical expectations. As for PG 1159-035, however, the observed value is largely in excess as compared to the theoretical predictions (see Costa et al. 1999). For DBV stars no detection has yet been made, but this situation could soon be changed by the ongoing observations of the hot DBV EC 20058 by Sullivan et al. (2004). In a recent paper, Winget et al. (2004) have called attention to the potential of employing DBV white dwarf stars to place constraints on the plasmon neutrino emissivity. Prompted by Winget et al. (2004)'s claims, we have computed in this work the change of period rate in pulsating DB white dwarf stars employing the new stellar models recently presented in Althaus \& Córsico (2004). These stellar models incorporate an updated input physics in addition to a self-consistent treatment of the time-dependent element diffusion to assess the shape of the chemical interfaces - an issue of fundamental importance in the context of white dwarf pulsations. In view of the lack of modern tabulations of $\dot{P} / P$ in DBV stars in the literature, we have included tables providing $P$ and $\dot{P} / P$ values corresponding to dipole modes $(\ell=1)$ for different stellar masses, effective temperatures and thickness of the helium envelope. We have also examined the effects of varying the neutrino emission rate on the $\dot{P} / P$ values. We find, in agreement with previous studies, that the rate of period change is a function mainly of the total stellar mass - there is more than a factor 6 between the $\dot{P} / P$ values for 0.50 - and $0.85-M_{\odot}$ models. At variance, the rates of period change are rather insensitive to the thickness of the helium envelope. We also find that, within the DBV instability strip, $\dot{P} / P$ is strongly dependent on the neutrino energy losses. Specifically, the $\dot{P} / P$ values are considerably smaller (by a factor of about 3-5) when neutrino losses are ignored in our models. Our results are consistent with the conclusion 


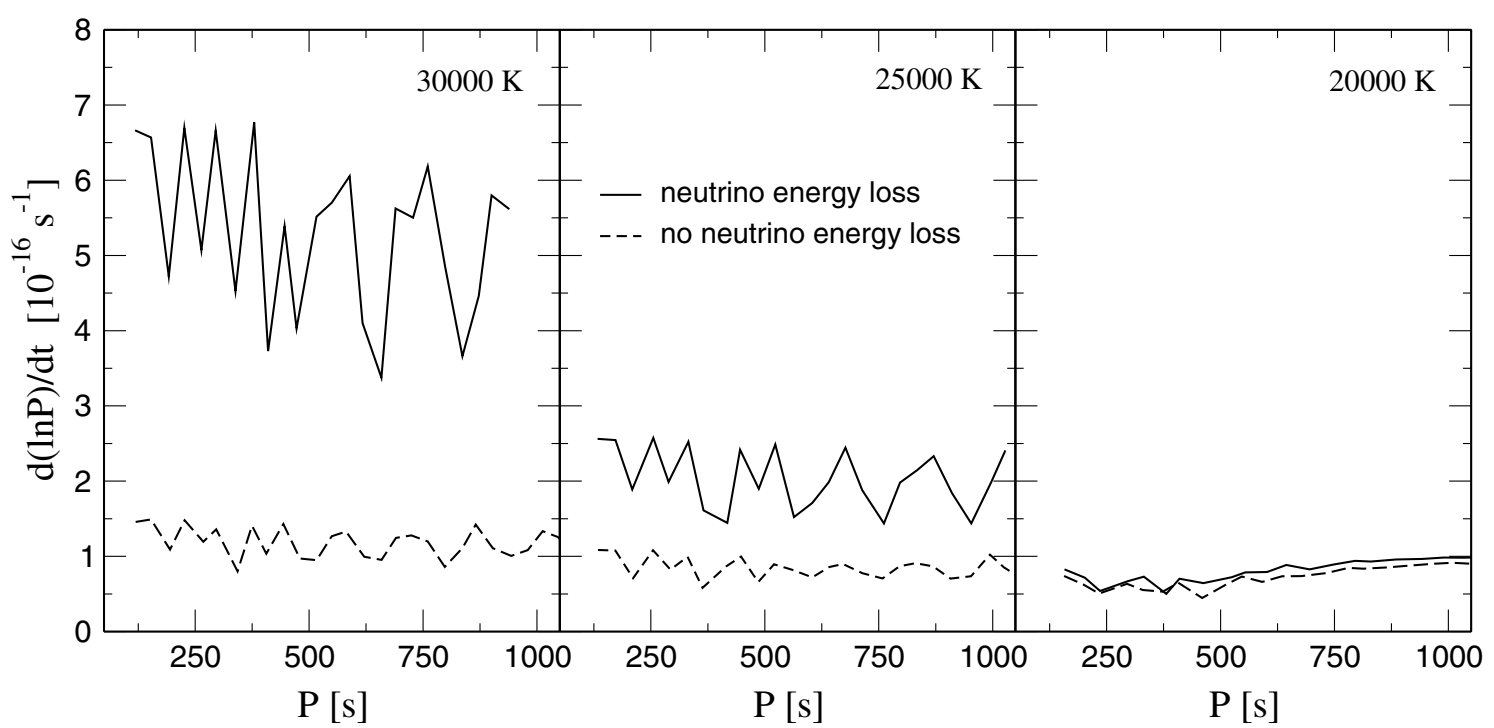

Fig. 5. The relative rate of period change in terms of the period corresponding to the same models as in Fig. 4, at effective temperatures of $\approx 30000, \approx 25000$ and $\approx 20000 \mathrm{~K}$. Continuous (dashed) lines correspond to computations including (ignoring) the effect of neutrinos.

arrived at recently by Winget et al. (2004) about the feasibility of quantitatively constraining the production rate of plasmon neutrinos by employing pulsating DB white dwarfs.

Acknowledgements. We wish to acknowledge the suggestions and comments of an anonymous referee that strongly improved the original version of this work. We warmly acknowledge E. García-Berro for a careful reading of the manuscript. This research was supported by the Instituto de Astrofísica La Plata. L.G.A. acknowledges the Spanish MCYT for a Ramón y Cajal Fellowship.

\section{References}

Althaus, L. G., \& Córsico, A. H. 2004, A\&A, 417, 1115

Althaus, L. G., Serenelli, A. M., Córsico, A. H., \& Montgomery, M. H. 2003, A\&A, 404, 593

Bradley, P. A. 1996, ApJ, 468, 350

Bradley, P. A., \& Winget, D. E. 1991, ApJS, 75, 463

Bradley, P. A., \& Winget, D. E. 1994, ApJ, 430, 850

Bradley, P. A., Winget, D. E., \& Wood, M. A. 1993, ApJ, 406, 661

Brassard, P., Fontaine, G., Wesemael, F., Kawaler, S. D., \& Tassoul, M. 1991, ApJ, 367, 601

Córsico, A. H., Althaus, L. G., Benvenuto, O. G., \& Serenelli, A. M. 2001b, A\&A, 380, L17

Córsico, A. H., Benvenuto, O. G., Althaus, L. G., Isern, J., \& García-Berro, E. 2001a, New Astron., 6, 197

Costa, J. E. S., Kepler, S. O., \& Winget, D. E. 1999, ApJ, 522, 973

D'Antona, F., \& Mazzitelli, I. 1991, in Evolution of Stars: the Photospheric Abundance Connection, ed. G. Michaud, \& A. Tutukov (Dordrecht: Kluwer), IAU Symp., 145, 399

Dehner, B. T., \& Kawaler, S. D. 1995, ApJ, 445, L141

Gautschy, A., \& Althaus, L. G. 2002, A\&A, 382, 141

Herwig, F., Blöcker, T., Langer, N., \& Driebe, T. 1999, A\&A, 349, L5

Iben, I. Jr. 1989, in Evolution of Peculiar Red Giant Stars, ed. H. R. Johnson, \& B. Zuckerman (Cambridge: Cambridge Univ. Press), IAU Coll., 106, 205

Iben, I. Jr., Kaler, J. B., Truran, J. W., \& Renzini, A. 1983, ApJ, 264, 605

Iglesias, C. A., \& Rogers, F. J. 1996, ApJ, 464, 943

Isern, J., Hernanz, M., \& García-Berro, E. 1992, ApJ, 392, L23
Kawaler, S. D., \& Bradley, P. A. 1994, ApJ, 427, 415

Kawaler, S. D., Hansen, C. J., \& Winget, D. E. 1985a, ApJ, 295, 547

Kawaler, S. D., Winget, D. E., \& Hansen, C. J. 1985b, ApJ, 298, 752

Kawaler, S. D., Winget, D. E., Iben, I., Jr., \& Hansen, C. J. 1986, ApJ, 302,530

Kepler, S. O., Mukadam, A. S., Winget, D. E., et al. 2000, ApJ, 534, L185

Kepler, S. O., et al. 1991, ApJ, 378, L45

Magni, G., \& Mazzitelli, I. 1979, A\&A, 72, 134

McGraw, J. T., Liebert, J., Starrfield, S. G., \& Green, R. 1979, in White Dwarfs and Variable Degenerate Stars, ed. H. M. Van Horn, \& V. Weidemann (Rochester: University of Rochester), IAU Coll., 53, 377

Mestel, L. 1952, MNRAS, 112, 583

Metcalfe, T., Montgomery, M. H., \& Kawaler, S. D. 2003, MNRAS, 344, L88

Metcalfe, T. S., Nather, R. E., \& Winget, D. E. 2000, ApJ, 545, 974

Metcalfe, T. S., Winget, D. E., \& Charbonneau, P. 2001, ApJ, 557, 1021

Metcalfe, T. S., Salaris, M., \& Winget, D. E. 2002, ApJ, 573, 803

Mukadam, A. S., Kepler, S. O., Winget, D. E., et al. 2003, ApJ, 594, 961

O’Brien, M. S., \& Kawaler, S. D. 2000, ApJ, 539, 372

Salaris, M., Domínguez, I., García-Berro, E., et al. 1997, ApJ, 486, 413

Schönberner, D. 1979, A\&A, 79, 108

Sullivan, D. J., et al. (the WET collaboration) 2004, in preparation

Tassoul, M., Fontaine, G., \& Winget, D. E. 1990, ApJS, 72, 335

Werner, K. 2001, Ap\&SS, 275, 27

Winget, D. E., Van Horn, H. M., Tassoul, M., et al. 1982a, ApJ, 252, L65

Winget, D. E., Robinson, E. L., Nather, R. E., \& Fontaine, G. 1982b, ApJ, 262, L11

Winget, D. E., Hansen, C. J., \& Van Horn, H. M. 1983, Nature, 303, 781

Winget, D. E., Robinson, E. L., Nather, R. E., Kepler, S. O., \& O'Donoghue, D. 1985, ApJ, 292, 606

Winget, D. E., Hansen, C. J., Liebert, J., et al. 1987, ApJ, 315, L77

Winget, D. E., Sullivan, D. J., Metcalfe, T. S., Kawaler, S. D., \& Montgomery, M. H. 2004, ApJ, 602, L109 